\title{
An efficient technique based on cubic B-spline functions for solving time-fractional advection diffusion equation involving Atangana-Baleanu derivative
}

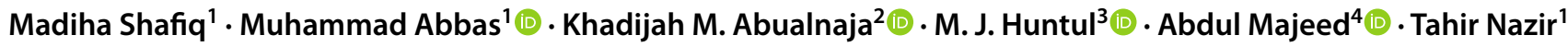

Received: 9 June 2021 / Accepted: 18 July 2021 / Published online: 4 August 2021

○) The Author(s), under exclusive licence to Springer-Verlag London Ltd., part of Springer Nature 2021

\begin{abstract}
The present paper deals with cubic B-spline approximation together with $\theta$-weighted scheme to obtain numerical solution of the time fractional advection diffusion equation using Atangana-Baleanu derivative. To discretize the Atangana-Baleanu time derivative containing a non-singular kernel, finite difference scheme is utilized. The cubic basis functions are associated with spatial discretization. The current discretization scheme used in the present study is unconditionally stable and the convergence is of order $O\left(h^{2}+\Delta t^{2}\right)$. The proposed scheme is validated through some numerical examples which reveal the current scheme is feasible and quite accurate.
\end{abstract}

Keywords Advection diffusion equation · Cubic B-spline functions · Spline interpolation · Atangana-Baleanu time fractional derivative $\cdot$ Finite difference formulation $\cdot$ Stability and convergence

\section{Introduction}

The calculus of derivatives and integrals of non-integer order (that is fractional order) is not a new concept. Its footprints of inspiration span three decades and has multidimensional

Madiha Shafiq

madihashafiq007@gmail.com

$\triangle$ Muhammad Abbas

muhammad.abbas@uos.edu.pk

$\triangle$ Khadijah M. Abualnaja

Kh.abualnaja@tu.edu.sa

M. J. Huntul

mhantool@jazanu.edu.sa

Abdul Majeed

abdulmajeed@ue.edu.pk

1 Department of Mathematics, University of Sargodha, Sargodha 40100, Pakistan

2 Department of Mathematics and Statistics, College of Science, Taif University, P. O. Box 11099, Taif 21944, Saudi Arabia

3 Department of Mathematics, Faculty of Science, Jazan University, Jazan, Saudi Arabia

4 Department of Mathematics, Division of Science and Technology, University of Education, Lahore 54770, Pakistan patterns of its practical implementations in science and engineering. This connection to other sciences makes fractional calculus more vital and indispensable. The fractional calculus has a long track of exertion in various theories and their applications. For example, it has been successfully used in viscoplastic and viscoelastic flow [1], transport problems [2], control theory [3], tumor development [4], continuum mechanics [5], random walks [6] and turbulence [7, 8]. In contrary to the conventional integer order counterparts, the fractional models which are described by fractional differential or integral equations possess more reliable and certain characteristics and thus behave more appropriately. Consequently, there is a growing need to explore solution techniques to study these models. More often, the analytical solution of most of the fractional differential equations cannot be acquired. Therefore, the search for approximate and analytical approaches is a subject matter of many lately published research papers. In this paper, approximate solution of the following time fractional advection diffusion equation (TFADE) shall be studied:

$$
\begin{aligned}
\frac{\partial^{\gamma} w(r, t)}{\partial t^{\gamma}}= & \Phi \frac{\partial^{2} w(r, t)}{\partial r^{2}} \\
& -\Psi \frac{\partial w(r, t)}{\partial r}+q(r, t), \quad r \in[a, b], t \in\left[t_{0}, T\right]
\end{aligned}
$$


with initial condition:

$w\left(r, t_{0}\right)=\phi(r)$

and the boundary conditions:

$w(a, t)=\psi_{1}(t), \quad w(b, t)=\psi_{2}(t)$,

where $\gamma \in(0,1)$ and $q(r, t)$ is the source term. $\Phi>0$ and $\Psi$ are the diffusion coefficient and average velocity, respectively. $\frac{\partial^{\gamma}}{\partial t^{\gamma}} w(r, t)$ is the Atangana-Baleanu fractional derivative (ABFD) based on non-singular kernel. The ABFD contains the Mittag-Leffler function that is the cornerstone of the fractional calculus. Applications of the Atangana-Baleanu operator have been explored in fields as diverse as heat transfer [9], chaos theory [10], oscillators [11], the hepatitis E virus model [12], cancer chemotherapy [13], smoking model [14] and coronavirus [15].

As noted in the literature, significant work has been done on numerical and analytical solutions of the TFADE. Mardani et al. [16] presented a meshless scheme based on the moving least square approximation for solving the TFADE with variable coefficients involving Caputo derivative. Bu et al. [17] proposed a V-cycle multigrid method for multi-term TFADEs. Sarboland [18] employed a meshfree approach for approximate solution of the time fractional partial differential equations based on multiquadric quasiinterpolation operator. The polynomial spectral collocation scheme has been constructed by Tian et al. [19] for solving the space fractional advection diffusion equation (FADE). Zheng et al. [20] studied the space FADE by means of a finite element method. An explicit and an implicit difference approximations have been developed by Shen et al. [21] for the solution of space-time Riesz-Caputo FADE. Azin et al. [22] established a hybrid method based on the modified Legendre and the Chebyshev cardinal functions for solving TFADE. The analytical and numerical investigations of twodimensional TFADE have been carried out by Ahmed et al. [23] involving memory and a concentrated source.

Mirza and Vieru [24] obtained the fundamental solutions of TFADE by employing the Laplace and Fourier transforms. Baleanu et al. [25] presented the variational homotopic perturbation and q-homotopic analysis methods for solving the fractional advection differential equation involving Caputo and Caputo-Fabrizio derivatives. Analytical solutions for FADE have been obtained by Rubbab et al. [26] utilizing the integral transforms technique based on time-dependent pulses on the boundary. Rubbab et al. [27] developed the finite difference and pseudo-spectral collocation schemes and analysed the numerical simulation of FADE with Caputo-Fabrizio operator in cylindrical domains. Korpinar et al. [28] presented the fractional homotopy perturbation transform scheme for the time fractional Gardner equation involving ABFD with Mittag-Leffler kernel. Owolabi [29, 30] proposed computational schemes for time fractional Kuramoto-Sivashinsky equation and multi-component systems with ABFD, respectively. A computational algorithm based on homotopic method has been employed by Kumar et al. [31] to investigate the fractional vibration equation. Hosseininia and Heydari [32] established a meshfree scheme by utilizing the moving least squares shape functions for the numerical solution of nonlinear 2D telegraph equation with variable-order ABFD. Inc et al. [33] analysed the logarithmic-KdV equation with Atangana-Baleanu operator. Bas and Ozarslan [34] obtained analytical solutions of fractional models (population growth, blood alcohol model, logistic equation, Newton's law of cooling) involving ABFD using Laplace transform. Akgül [35] presented a novel technique for investigation of fractional differential equations involving the ABFD. Akgül and Modanli [36] proposed the Crank-Nicholson difference scheme method for the third order fractional partial differential equation with ABFD. Attia et al. [37] developed the computational approach to find an approximate solution of the TFADE.

In recent years, several authors worked on numerous numerical procedures using B-spline interpolation for solving fractional differential equations. In particular, due to rich geometrical features of the spline functions, they are found to be a powerful tool in curve approximation. Yaseen et al. [38] presented a computational method for the generalized nonlinear time-fractional Klein-Gordon equation involving Caputo operator via cubic trigonometric B-spline functions. Abbas et al. [39] proposed the new cubic B-spline approximation based method for numerical solution of non-linear third order Korteweg-de Vries equation. A redefined cubic B-spline functions based scheme has been developed by Khalid et al. [40] for numerical solution of time fractional Allen-Cahn equation. The authors in [41, 42] derived numerical algorithms from the extended cubic B-spline functions to investigate the computational solutions for the time-fractional fisher and telegraph equations. Iqbal et al. [43] investigated numerical simulation of Kuramoto-Sivashinsky equation using new quintic polynomial B-spline functions. Khalid et al. [44] presented a computational method using redefined extended B-spline approximation for solving TFADE.

This paper presents the numerical scheme for the TFADE based on CBS functions. Current proposed method used the $\theta$-weighted scheme and Atangana-Baleanu operator. The usage of non-singular kernel operator in B-spline methods is novel. The stability and convergence analysis of the scheme is also performed to avoid any false result. To the best of the author's knowledge, the proposed algorithm for TFADE is novel and it has not been studied yet. 
The current paper is structured in the following pattern: In Sect. 2, the ABFD, Parseval's identity and basis functions are defined and currently developed scheme is presented in Sect. 3. Sections 4 and 5 contain the stability of algorithm and analysis of convergence, respectively. The validity and efficiency of the proposed method is analysed in Sect. 6 and finally, the conclusion is given in Sect. 7.
Now, we suppose that $W(r, t)$ be the CBS approximation for $w(r, t)$ s.t.

$W(r, t)=\sum_{k=-1}^{N+1} \mu_{k}^{m}(t) C_{k}(r)$,

where $\mu_{k}^{m}(t)$, the control points, are to be computed at each time level and $C_{k}(r)$, the CBS functions, are defined as

$C_{k}(r)$

$$
=\frac{1}{6 h^{3}} \begin{cases}\left(r-r_{k-2}\right)^{3}, & \text { if } r \in\left[r_{k-2}, r_{k-1}\right), \\ h^{3}+3 h^{2}\left(r-r_{k-1}\right)+3 h\left(r-r_{k-1}\right)^{2}-3\left(r-r_{k-1}\right)^{3}, & \text { if } r \in\left[r_{k-1}, r_{k}\right), \\ h^{3}+3 h^{2}\left(r_{k+1}-r\right)+3 h\left(r_{k+1}-r\right)^{2}-3\left(r_{k+1}-r\right)^{3}, & \text { if } r \in\left[r_{k}, r_{k+1}\right), \\ \left(r_{k+2}-r\right)^{3}, & \text { if } r \in\left[r_{k+1}, r_{k+2}\right), \\ 0, & \text { otherwise. }\end{cases}
$$

\section{Preliminaries}

Definition 1 The Atangana-Baleanu fractional derivative (ABFD) $\frac{\partial^{\gamma}}{\partial t t^{\gamma}} w(r, t)$ without singular kernel of order $\gamma \in(0,1)$ is given by

$\frac{\partial^{\gamma}}{\partial t^{\gamma}} w(r, t)=\frac{R(\gamma)}{1-\gamma} \int_{0}^{t} \frac{\partial}{\partial v} w(r, v) E_{\gamma}\left[-\frac{\gamma}{1-\gamma}(t-v)^{\gamma}\right] \mathrm{d} v$,

where $R(\gamma)$ is a normalization function which satisfies $R(0)=R(1)=1 . E_{\gamma, \sigma}(r)$ is the Mittag-Leffler function satisfying $E_{\gamma, 1}(r)=E_{\gamma}(r)$, which is defined as

$E_{\gamma, \sigma}(r)=\sum_{p=0}^{\infty} \frac{r^{p}}{\Gamma(\gamma p+\sigma)}$.

Definition 2 If $\tilde{f} \in L^{2}[a, b]$, then Parseval's identity is given as [45]:

$\sum_{n=-\infty}^{\infty}|\hat{f}(n)|^{2}=\int_{a}^{b}|\tilde{f}(r)|^{2} \mathrm{~d} r$,

where $\hat{f}(n)=\int_{a}^{b} \tilde{f}(r) e^{2 \pi i n r} \mathrm{~d} r$ for every integer $n$ is its Fourier transform.

\subsection{Basis functions}

The spatial domain $[a, b]$ such that $a=r_{0}<r_{1}<\cdots<r_{N}=b$, where $r_{k}=r_{0}+k h, k=0(1) N$, be divided into $N$ subintervals of equal length $h=\frac{b-a}{N}$.
The CBS functions retain abundant supply of geometrical properties, such as convex hull property, local support, geometric invariability, symmetry, non-negativity and the partition of unity [40]. Furthermore, $C_{-1}, C_{0}, \ldots, C_{N+1}$ have been organized in that manner that they can serve as basis for space of all third degree splines. The relations (2.3) and (2.4) yield the following approximations:

$\left\{\begin{array}{l}(W)_{k}^{m}=\left(\frac{1}{6}\right) \mu_{k-1}^{m}+\left(\frac{4}{6}\right) \mu_{k}^{m}+\left(\frac{1}{6}\right) \mu_{k+1}^{m}, \\ \left(W_{r}\right)_{k}^{m}=\left(\frac{1}{2 h}\right) \mu_{k+1}^{m}+\left(-\frac{1}{2 h}\right) \mu_{k-1}^{m}, \\ \left(W_{r r}\right)_{k}^{m}=\left(\frac{1}{h^{2}}\right) \mu_{k-1}^{m}+\left(-\frac{2}{h^{2}}\right) \mu_{k}^{m}+\left(\frac{1}{h^{2}}\right) \mu_{k+1}^{m} .\end{array}\right.$

\section{Illustration of the scheme}

Let the time domain $[0, T]$ using the knots $0=t_{0}<t_{1}<\cdots<t_{M}=T$, where $t_{m}=m \Delta t$ and $m=0,1, \ldots, M$, be divided into $M$ equal subintervals of the length $\Delta t=\frac{T}{M}$. The ABFD involved in (1.1) is discretized at $t=t_{m+1}$ as

$$
\begin{aligned}
& \frac{\partial^{\gamma}}{\partial t^{\gamma}} w\left(r, t_{m+1}\right) \\
& \quad=\frac{R(\gamma)}{1-\gamma} \int_{0}^{t_{m+1}} \frac{\partial}{\partial v} w(r, v) E_{\gamma} \\
& \quad\left[-\frac{\gamma}{1-\gamma}\left(t_{m+1}-v\right)^{\gamma}\right] d v, \quad 0<\gamma<1, \\
& \quad=\frac{R(\gamma)}{1-\gamma} \sum_{s=0}^{m} \int_{t_{s}}^{t_{s+1}} \frac{\partial}{\partial v} w(r, v) E_{\gamma}\left[-\frac{\gamma}{1-\gamma}\left(t_{m+1}-v\right)^{\gamma}\right] \mathrm{d} v .
\end{aligned}
$$


Utilizing forward difference formulation, Eq. (3.1) becomes

$$
\begin{aligned}
& \frac{\partial^{\gamma}}{\partial t^{\gamma}} w\left(r, t_{m+1}\right) \\
& \quad=\frac{R(\gamma)}{1-\gamma} \sum_{s=0}^{m} \frac{w\left(r, t_{s+1}\right)-w\left(r, t_{s}\right)}{\Delta t} \int_{t_{s}}^{t_{s+1}} E_{\gamma} \\
& \quad\left[-\frac{\gamma}{1-\gamma}\left(t_{m+1}-v\right)^{\gamma}\right] \mathrm{d} v+\lambda_{\Delta t}^{m+1} \\
& =\frac{R(\gamma)}{1-\gamma} \sum_{s=0}^{m}\left[w\left(r, t_{m-s+1}\right)-w\left(r, t_{m-s}\right)\right]\left\{(s+1) E_{\gamma, 2}\right. \\
& \left.\quad-\frac{\gamma}{1-\gamma}((s+1) \Delta t)^{\gamma}\right] \\
& \left.-s E_{\gamma, 2}\left[-\frac{\gamma}{1-\gamma}(s \Delta t)^{\gamma}\right]\right\}+\lambda_{\Delta t}^{m+1} \\
& =\frac{R(\gamma)}{1-\gamma} \sum_{s=0}^{m}\left[w\left(r, t_{m-s+1}\right)-w\left(r, t_{m-s}\right)\right] \\
& \left((s+1) E_{s+1}-s E_{s}\right)+\lambda_{\Delta t}^{m+1} .
\end{aligned}
$$

Hence

$$
\frac{\partial^{\gamma}}{\partial t^{\gamma}} w\left(r, t_{m+1}\right)=\frac{R(\gamma)}{1-\gamma} \sum_{s=0}^{m} l_{s}\left[w\left(r, t_{m-s+1}\right)-w\left(r, t_{m-s}\right)\right]+\lambda_{\Delta t}^{m+1},
$$

where $E_{s}=E_{\gamma, 2}\left[-\frac{\gamma}{1-\gamma}(s \Delta t)^{\gamma}\right]$ and $l_{s}=(s+1) E_{s+1}-s E_{s}$. It is straightforward to observe that

- $l_{s}>0$ and $l_{0}=E_{1}, s=1: 1: m$,

- $l_{0}>l_{1}>l_{2}>\cdots>l_{s}, l_{s} \rightarrow 0$ as $s \rightarrow \infty$,

- $\sum_{s=0}^{m}\left(l_{s}-l_{s+1}\right)+l_{m+1}=\left(E_{1}-l_{1}\right)+\sum_{s=1}^{m-1}\left(l_{s}-l_{s+1}\right)$ $+l_{m}=E_{1}$.

Moreover, the truncation error $\lambda_{\Delta t}^{m+1}$ is given by [46]:

$$
\begin{aligned}
\lambda_{\Delta t}^{m+1}= & \frac{R(\gamma)}{1-\gamma} \sum_{s=0}^{m} \int_{t_{s}}^{t_{s+1}} \frac{\Delta t}{2} \frac{\partial^{2} w\left(r, t_{s}\right)}{\partial t^{2}} E_{\gamma}\left[-\frac{\gamma}{1-\gamma}\left(t_{m+1}-v\right)^{\gamma}\right] \mathrm{d} v \\
= & \frac{R(\gamma)}{1-\gamma} \frac{(\Delta t)^{2}}{2} \sum_{s=0}^{m} \frac{\partial^{2} w\left(r, t_{s}\right)}{\partial t^{2}}\left\{(m-s+1) E_{\gamma, 2}\right. \\
& {\left[-\frac{\gamma}{1-\gamma}((m-s+1) \Delta t)^{\gamma}\right] } \\
& \left.-(m-s) E_{\gamma, 2}\left[-\frac{\gamma}{1-\gamma}((m-s) \Delta t)^{\gamma}\right]\right\} \\
= & \frac{R(\gamma)}{1-\gamma} \frac{(\Delta t)^{2}}{2} \sum_{s=0}^{m} \frac{\partial^{2} w\left(r, t_{s}\right)}{\partial t^{2}} \\
& \left((m-s+1) E_{m-s+1}-(m-s) E_{m-s}\right) \\
\leq & \frac{R(\gamma)}{1-\gamma} \frac{(\Delta t)^{2}}{2}\left[\max _{0 \leq t \leq t_{m}} \frac{\partial^{2} w(r, t)}{\partial t^{2}}\right] c_{1},
\end{aligned}
$$

where $c_{1}$ is a constant:

$\left|\lambda_{\Delta t}^{m+1}\right| \leq \vartheta(\Delta t)^{2}$

where $\vartheta$ is a constant.

Using (3.2) and $\theta$-weighted scheme then Eq. (1.1) takes the following form:

$$
\begin{aligned}
& \frac{R(\gamma)}{1-\gamma} \sum_{s=0}^{m} l_{s}\left[w\left(r, t_{m-s+1}\right)-w\left(r, t_{m-s}\right)\right] \\
& =\theta\left(\Phi w_{r r}\left(r, t_{m+1}\right)-\Psi w_{r}\left(r, t_{m+1}\right)\right) \\
& \quad+(1-\theta)\left(\Phi w_{r r}\left(r, t_{m}\right)-\Psi w_{r}\left(r, t_{m}\right)\right)+q\left(r, t_{m+1}\right) .
\end{aligned}
$$

Discretizing (3.4) along spatial direction for $\theta=1$, we acquire

$$
\begin{aligned}
& E_{1} w_{k}^{m+1}-\xi \Phi\left(w_{r r}\right)_{k}^{m+1}+\xi \Psi\left(w_{r}\right)_{k}^{m+1} \\
& =E_{1} w_{k}^{m}-\sum_{s=1}^{m} l_{s}\left(w_{k}^{m-s+1}-w_{k}^{m-s}\right)+\xi q_{k}^{m+1},
\end{aligned}
$$

where $\xi=\frac{1-\gamma}{R(\gamma)}, w_{k}^{m}=w\left(r_{k}, t_{m}\right)$ and $q_{k}^{m+1}=q\left(r_{k}, t_{m+1}\right)$.

Using (2.5) in (3.5), we get

$$
\begin{gathered}
\left(E_{1} b_{1}-\xi \Phi b_{4}-\xi \Psi b_{3}\right) \mu_{k-1}^{m+1} \\
\quad+\left(E_{1} b_{2}-\xi \Phi b_{5}\right) \mu_{k}^{m+1}+\left(E_{1} b_{1}-\xi \Phi b_{4}+\xi \Psi b_{3}\right) \mu_{k+1}^{m+1} \\
=E_{1}\left(b_{1} \mu_{k-1}^{m}+b_{2} \mu_{k}^{m}+b_{1} \mu_{k+1}^{m}\right) \\
\quad-\sum_{s=1}^{m} l_{s}\left[b_{1}\left(\mu_{k-1}^{m-s+1}-\mu_{k-1}^{m-s}\right)+b_{2}\left(\mu_{k}^{m-s+1}-\mu_{k}^{m-s}\right)\right. \\
\left.\quad+b_{1}\left(\mu_{k+1}^{m-s+1}-\mu_{k+1}^{m-s}\right)\right]+\xi q_{k}^{m+1},
\end{gathered}
$$

where $b_{1}=\frac{1}{6}, b_{2}=\frac{4}{6}, b_{3}=\frac{1}{2 h}, b_{4}=\frac{1}{h^{2}}, b_{5}=\frac{-2}{h^{2}}$ and $\mu_{k}^{m}=\mu_{k}\left(t^{m}\right)$.

The system (3.6) consists of $N+1$ linear equations in $N+3$ unknowns. For the unique solution, two additional equations can be achieved from the boundary conditions (1.3). Consequently, a matrix system of dimension $(N+3) \times(N+3)$ is obtained:

$A \mu^{m+1}=B\left(\sum_{s=0}^{m-1}\left(l_{s}-l_{s+1}\right) \mu^{m-s}+l_{m} \mu^{0}\right)+\xi J^{m+1}$,

where 


$$
A=\left(\begin{array}{ccccccc}
b_{1} & b_{2} & b_{1} & & & \\
y_{1} & y_{2} & y_{3} & & & \\
& y_{1} & y_{2} & y_{3} & & \\
& & \ddots & \ddots & \ddots & \\
& & & y_{1} & y_{2} & y_{3} \\
& & & & y_{1} & y_{2} & y_{3} \\
& & & & b_{1} & b_{2} & b_{1}
\end{array}\right),
$$$$
B=\left(\begin{array}{ccccccc}
0 & 0 & 0 & & & & \\
b_{1} & b_{2} & b_{1} & & & & \\
& b_{1} & b_{2} & b_{1} & & & \\
& & \ddots & \ddots & \ddots & & \\
& & & b_{1} & b_{2} & b_{1} & \\
& & & & b_{1} & b_{2} & b_{1} \\
& & & & 0 & 0 & 0
\end{array}\right) \text {, }
$$$$
\mu^{m}=\left(\begin{array}{c}
\mu_{-1}^{m} \\
\mu_{0}^{m} \\
\mu_{1}^{m} \\
\vdots \\
\mu_{N-1}^{m} \\
\mu_{N}^{m} \\
\mu_{N+1}^{m}
\end{array}\right) \text { and } J^{m+1}=\left(\begin{array}{c}
\psi_{1}^{m+1} \\
q_{0}^{m+1} \\
q_{1}^{m+1} \\
\vdots \\
q_{N-1}^{m+1} \\
q_{N}^{m+1} \\
\psi_{2}^{m+1}
\end{array}\right),
$$

where $y_{1}=E_{1} b_{1}-\xi \Phi b_{4}-\xi \Psi b_{3}, y_{2}=E_{1} b_{2}-\xi \Phi b_{5}$ and $y_{3}=E_{1} b_{1}-\xi \Phi b_{4}+\xi \Psi b_{3}$. Before employing (3.7), the initial vector $\mu^{0}=\left[\mu_{-1}^{0}, \mu_{0}^{0}, \ldots, \mu_{N+1}^{0}\right]^{T}$ is achieved by utilizing the initial conditions as

$$
\begin{cases}\left(W_{r}\right)_{k}^{0}=\phi^{\prime}\left(r_{k}\right), & k=0, \\ (W)_{k}^{0}=\phi\left(r_{k}\right), & k=0: 1: N, \\ \left(W_{r}\right)_{k}^{0}=\phi^{\prime}\left(r_{k}\right), & k=N .\end{cases}
$$

In matrix form, the above system of equations can be represented as

$G \mu^{0}=H$,

where

$G=\left(\begin{array}{ccccccc}-b_{3} & 0 & b_{3} & & & & \\ b_{1} & b_{2} & b_{1} & & & & \\ & b_{1} & b_{2} & b_{1} & & & \\ & & \ddots & \ddots & \ddots & & \\ & & & b_{1} & b_{2} & b_{1} & \\ & & & & b_{1} & b_{2} & b_{1} \\ & & & & -b_{3} & 0 & b_{3}\end{array}\right)$ and

$$
H=\left(\begin{array}{c}
\phi^{\prime}\left(r_{0}\right) \\
\phi\left(r_{0}\right) \\
\phi\left(r_{1}\right) \\
\vdots \\
\phi\left(r_{N-1}\right) \\
\phi\left(r_{N}\right) \\
\phi^{\prime}\left(r_{N}\right)
\end{array}\right)
$$

Equation (3.9) is easily solvable for $\mu^{0}$ by means of a suitable numerical algorithm. All the numerical computations are performed in Mathematica 12.

\section{The stability analysis}

During the computational procedure, when the error does not amplify, the numerical scheme is presumed to be stable [47]. Here, the presented scheme is analysed for stability by implementing the Fourier method [41, 44]. For this, suppose $\delta_{k}^{m}$ and $\tilde{\delta}_{k}^{m}$ symbolize the growth factor and its estimation in Fourier mode. The error $\chi_{k}^{m}$ can be presented as

$\chi_{k}^{m}=\delta_{k}^{m}-\tilde{\delta}_{k}^{m}, \quad m=0(1) M, k=1(1) N-1$.

For simplicity, we analyse the stability of the scheme given in (3.6) for force-free case $(q=0)$ only, so that from Eq. (3.6), we obtain

$$
\begin{gathered}
\left(E_{1} b_{1}-\xi \Phi b_{4}-\xi \Psi b_{3}\right) \chi_{k-1}^{m+1}+\left(E_{1} b_{2}-\xi \Phi b_{5}\right) \chi_{k}^{m+1} \\
+\left(E_{1} b_{1}-\xi \Phi b_{4}+\xi \Psi b_{3}\right) \chi_{k+1}^{m+1} \\
=E_{1}\left(b_{1} \chi_{k-1}^{m}+b_{2} \chi_{k}^{m}+b_{1} \chi_{k+1}^{m}\right) \\
\quad-\sum_{s=1}^{m} l_{s}\left[b_{1}\left(\chi_{k-1}^{m-s+1}-\chi_{k-1}^{m-s}\right)+b_{2}\left(\chi_{k}^{m-s+1}-\chi_{k}^{m-s}\right)\right. \\
\left.\quad+b_{1}\left(\chi_{k+1}^{m-s+1}-\chi_{k+1}^{m-s}\right)\right] .
\end{gathered}
$$

From initial and boundary conditions, we can write

$\chi_{k}^{0}=\phi\left(r_{k}\right), k=1: 1: N$

and

$\chi_{0}^{m}=\psi_{1}\left(t_{m}\right), \quad \chi_{N}^{m}=\psi_{2}\left(t_{m}\right), \quad m=0: 1: M$.

Define grid function as

$\chi^{m}= \begin{cases}\chi_{k}^{m}, & r_{k}-\frac{h}{2}<r \leq r_{k}+\frac{h}{2}, k=1(1) N-1, \\ 0, & a \leq r \leq a+\frac{h}{2} \text { or } b-\frac{h}{2} \leq r \leq b .\end{cases}$

The Fourier expansion for $\chi^{m}(r)$ can be represented as

$\chi^{m}(r)=\sum_{n=-\infty}^{\infty} \zeta^{m}(n) \mathrm{e}^{\frac{2 \pi z n r}{b-a}}$,

where

$$
\begin{aligned}
& \zeta^{m}(n)=\frac{1}{b-a} \int_{a}^{b} \chi^{m}(r) \mathrm{e}^{\frac{-2 \pi z n r}{b-a}} \mathrm{~d} r, \\
& m=0: 1: M \text { and } \chi^{m}=\left[\chi_{1}^{m}, \chi_{2}^{m}, \ldots, \chi_{N-1}^{m}\right]^{T} .
\end{aligned}
$$

Applying $\|.\|_{2}$ norm, we acquire 


$$
\begin{aligned}
\left\|\chi^{m}\right\|_{2}= & \sqrt{h \sum_{k=1}^{N-1}\left|\chi_{k}^{m}\right|^{2}} \\
= & \left(\int_{a}^{a+\frac{h}{2}}\left|\chi^{m}\right|^{2} \mathrm{~d} r\right. \\
& \left.+\sum_{k=1}^{N-1} \int_{r_{k}-\frac{h}{2}}^{r_{k}+\frac{h}{2}}\left|\chi^{m}\right|^{2} \mathrm{~d} r+\int_{b-\frac{h}{2}}^{b}\left|\chi^{m}\right|^{2} \mathrm{~d} r\right)^{\frac{1}{2}} \\
= & \left(\int_{a}^{b}\left|\chi^{m}\right|^{2} \mathrm{~d} r\right)^{\frac{1}{2}} .
\end{aligned}
$$

By Parseval's identity (2.2), we have [45]

$$
\int_{a}^{b}\left|\chi^{m}\right|^{2} \mathrm{~d} r=\sum_{n=-\infty}^{\infty}\left|\zeta^{m}(n)\right|^{2} .
$$

Hence, we acquire

$\left\|\chi^{m}\right\|_{2}^{2}=\sum_{n=-\infty}^{\infty}\left|\zeta^{m}(n)\right|^{2}$.

Assume that (4.1)-(4.3) possess a solution in Fourier form as:

$\chi_{k}^{m}=\zeta^{m} \mathrm{e}^{i \alpha k h}$,

where $\alpha$ is a real number and $i=\sqrt{-1}$. Substituting (4.8) in (4.1) and simplifying, we acquire

$$
\begin{aligned}
& \left(E_{1} b_{1}-\xi \Phi b_{4}-\xi \Psi b_{3}\right) \zeta^{m+1} \mathrm{e}^{-i \alpha h}+\left(E_{1} b_{2}-\xi \Phi b_{5}\right) \zeta^{m+1} \\
& \quad+\left(E_{1} b_{1}-\xi \Phi b_{4}+\xi \Psi b_{3}\right) \zeta^{m+1} \mathrm{e}^{i \alpha h} \\
& \quad=\left(E_{1} b_{1} \zeta^{m} \mathrm{e}^{-i \alpha h}+E_{1} b_{2} \zeta^{m}+E_{1} b_{1} \zeta^{m} \mathrm{e}^{i \alpha h}\right) \\
& \quad-\sum_{s=1}^{m} l_{s}\left[b_{1}\left(\zeta^{m-s+1} \mathrm{e}^{-i \alpha h}-\zeta^{m-s} \mathrm{e}^{-i \alpha h}\right)\right. \\
& \quad+b_{2}\left(\zeta^{m-s+1}-\zeta^{m-s}\right) \\
& \left.\quad+b_{1}\left(\zeta^{m-s+1} \mathrm{e}^{i \alpha h}-\zeta^{m-s} \mathrm{e}^{i \alpha h}\right)\right]
\end{aligned}
$$

Utilizing the relation $\mathrm{e}^{i \alpha h}+\mathrm{e}^{-i \alpha h}=2 \cos (\alpha h)$ and assembling the identical terms, we acquire the following equality:

$\zeta^{m+1}=\frac{1}{\epsilon}\left[\zeta^{m}-\frac{1}{E_{1}} \sum_{s=1}^{m} l_{s}\left(\zeta^{m-s+1}-\zeta^{m-s}\right)\right]$,

where $\epsilon=1+\frac{12 \xi \Phi \sin ^{2}(\alpha h / 2)+3 \xi \Psi k h \sin \alpha h}{h^{2} E_{1}\left(3-2 \sin ^{2}(\alpha h / 2)\right)}$. Obviously $\epsilon \geq 1$ and $E_{1}>0$.
Lemma 4.1 If $\zeta^{m}$ is the solution for equation (4.10), then $\left|\zeta^{m}\right| \leq\left|\zeta^{0}\right|, \quad m=0,1,2, \cdots, M$.

Proof Mathematical induction will be availed. For $m=0$, (4.10) gives

$\left|\zeta^{1}\right|=\frac{1}{\epsilon}\left|\zeta^{0}\right| \leq\left|\zeta^{0}\right|, \quad \epsilon \geq 1$.

Assume that $\left|\zeta^{m}\right| \leq\left|\zeta^{0}\right|$ for $m=1,2, \ldots, M-1$, then

$$
\begin{aligned}
\left|\zeta^{m+1}\right| & \leq \frac{1}{\epsilon}\left|\zeta^{m}\right|-\frac{1}{\epsilon E_{1}} \sum_{s=1}^{m} l_{s}\left(\left|\zeta^{m-s+1}\right|-\left|\zeta^{m-s}\right|\right) \\
& \leq \frac{1}{\epsilon}\left|\zeta^{0}\right|-\frac{1}{\epsilon E_{1}} \sum_{s=1}^{m} l_{s}\left(\left|\zeta^{0}\right|-\left|\zeta^{0}\right|\right) \\
& \leq\left|\zeta^{0}\right| .
\end{aligned}
$$

Theorem 1 The present scheme (3.6) is unconditionally stable.

Proof Utilizing Lemma 4.1 and the expression (4.7), we acquire

$\left\|\chi^{m}\right\|_{2} \leq\left|\chi^{0}\right|_{2}, \quad \forall m=0,1, \ldots, M$.

Hence, the proposed numerical method is stable unconditionally.

\section{The convergence analysis}

We follow the procedure employed in [48] to study the convergence of the propounded method. First of all, the following theorem is brought forward $[49,50]$.

Theorem 2 Suppose that $w(r, t), q$ belong to $C^{4}[a, b]$ and $C^{2}[a, b]$, respectively, and $\Upsilon=\left\{a=r_{0}, r_{1}, \ldots, r_{N}=b\right\}$ be the partition of $[a, b]$ s.t. $r_{k}=a+k h, k=0,1, \ldots, N$. Let $\tilde{W}(r, t)$ be the unique spline that interpolates the solution curve at knots $r_{k} \in \Upsilon$, then there is a constant $\varrho_{k}$ not depending on $h$, then for every $t \geq 0$, we acquire

$\left\|D^{k}(w(r, t)-\tilde{W}(r, t))\right\|_{\infty} \leq \varrho_{k} h^{4-k}, k=0,1,2$.

Lemma 5.1 The CBS set $\left\{C_{-1}, C_{0}, \ldots, C_{N+1}\right\}$ presented in (2.4) satisfies the inequality

$\sum_{k=-1}^{N+1}\left|C_{k}(r)\right| \leq \frac{5}{3}, \quad 0 \leq r \leq 1$. 
Proof Employing the triangular inequality, we are able to write

$\left|\sum_{k=-1}^{N+1} C_{k}(r)\right| \leq \sum_{k=-1}^{N+1}\left|C_{k}(r)\right|$

At any knot $r_{k}$, we achieve

$$
\begin{aligned}
\sum_{k=-1}^{N+1}\left|C_{k}(r)\right| & =\left|C_{k-1}\left(r_{k}\right)\right|+\left|C_{k}\left(r_{k}\right)\right|+\left|C_{k+1}\left(r_{k}\right)\right| \\
& =\frac{1}{6}+\frac{4}{6}+\frac{1}{6}=1<\frac{5}{3} .
\end{aligned}
$$

Moreover, for $r \in\left[r_{k}, r_{k+1}\right]$, we get

$$
\begin{array}{rlrl}
\left|C_{k}(r)\right| & \leq \frac{4}{6} \quad \text { \&and } & \left|C_{k-1}(r)\right| \leq \frac{1}{6}, \\
\left|C_{k+1}(r)\right| \leq \frac{4}{6} \quad \text { \&and } & \left|C_{k+2}(r)\right| \leq \frac{1}{6} .
\end{array}
$$

Thus, for any point $r_{k} \leq r \leq r_{k+1}$, we obtain

$$
\sum_{k=-1}^{N+1}\left|C_{k}(r)\right|=\left|C_{k-1}(r)\right|+\left|C_{k}(r)\right|+\left|C_{k+1}(r)\right|+\left|C_{k+2}(r)\right| \leq \frac{5}{3} .
$$

Theorem 3 The computational approximation $W(r, t)$ to the analytical solution $w(r, t)$ for TFADE (1.1)-(1.3) exists. Furthermore, if $q$ belongs to $C^{2}[0,1]$, then

$\|w(r, t)-W(r, t)\|_{\infty} \leq \widetilde{\rho} h^{2}, \quad \forall t \geq 0$,

where $h$ is appropriately small and $\widetilde{\rho}>0$ is free of $h$.

Proof We assume that $\tilde{W}(r, t)=\sum_{k=-1}^{N+1} u_{k}^{m}(t) C_{k}(r)$ be the approximated spline for $W(r, t)$. By means of triangular inequality, we acquire

$\|w(r, t)-W(r, t)\|_{\infty} \leq\|w(r, t)-\tilde{W}(r, t)\|_{\infty}+\|\tilde{W}(r, t)-W(r, t)\|_{\infty}$.

With the aid of Theorem 2, we achieve

$\|w(r, t)-W(r, t)\|_{\infty} \leq \varrho_{0} h^{4}+\|\tilde{W}(r, t)-W(r, t)\|_{\infty}$.

The collocation conditions of proposed scheme are $L w\left(r_{k}, t\right)=L W\left(r_{k}, t\right)=q\left(r_{k}, t\right), k=0(1) N$. Suppose that

$L \tilde{W}(r, t)=\tilde{q}\left(r_{k}, t\right), \quad k=0(1) N$.

Thus, the difference $L\left(\tilde{W}\left(r_{k}, t\right)-W\left(r_{k}, t\right)\right)$ for any time stage $\mathrm{m}$ can be stated as

$$
\begin{aligned}
& \left(E_{1} b_{1}-\xi \Phi b_{4}-\xi \Psi b_{3}\right) \Omega_{k-1}^{m+1} \\
& \quad+\left(E_{1} b_{2}-\xi \Phi b_{5}\right) \Omega_{k}^{m+1}+\left(E_{1} b_{1}-\xi \Phi b_{4}+\xi \Psi b_{3}\right) \Omega_{k+1}^{m+1} \\
& =\left(E_{1} b_{1} \Omega_{k-1}^{m}+E_{1} b_{2} \Omega_{k}^{m}+E_{1} b_{1} \Omega_{k+1}^{m}\right)+\frac{\xi}{h^{2}} \rho_{k}^{m+1} \\
& \quad-\sum_{s=1}^{m} l_{s}\left[b_{1}\left(\Omega_{k-1}^{m-s+1}-\Omega_{k-1}^{m-s}\right)+b_{2}\left(\Omega_{k}^{m-s+1}-\Omega_{k}^{m-s}\right)\right. \\
& \left.\quad+b_{1}\left(\Omega_{k+1}^{m-s+1}-\Omega_{k+1}^{m-s}\right)\right] .
\end{aligned}
$$

The boundary conditions can be described as

$b_{1} \Omega_{k-1}^{m+1}+b_{2} \Omega_{k}^{m+1}+b_{1} \Omega_{k+1}^{m+1}=0, k=0, N$,

where

$\Omega_{k}^{m}=\mu_{k}^{m}-u_{k}^{m}, k=-1: 0: N+1$

and

$\rho_{k}^{m}=h^{2}\left[q_{k}^{m}-\tilde{q}_{k}^{m}\right], \quad k=0: 1: N$.

It is obvious from inequality (5.1) that

$\left|\rho_{k}^{m}\right|=h^{2}\left|q_{k}^{m}-\tilde{q}_{k}^{m}\right| \leq \rho h^{4}$.

Define $\quad \rho^{m}=\max \left\{\left|\rho_{k}^{m}\right| ; 0 \leq k \leq N\right\}, \quad e_{k}^{m}=\left|\Omega_{k}^{m}\right| \quad$ and $e^{m}=\max \left\{\left|e_{k}^{m}\right| ; 0 \leq k \leq N\right\}$.

When $m=0$, Eq. (5.5) becomes

$$
\begin{aligned}
\left(E_{1} b_{1}-\xi \Phi b_{4}-\xi \Psi b_{3}\right) \Omega_{k-1}^{1} & \\
& +\left(E_{1} b_{2}-\xi \Phi b_{5}\right) \Omega_{k}^{1}+\left(E_{1} b_{1}-\xi \Phi b_{4}+\xi \Psi b_{3}\right) \Omega_{k+1}^{1} \\
& =\left(E_{1} b_{1} \Omega_{k-1}^{0}+E_{1} b_{2} \Omega_{k}^{0}+E_{1} b_{1} \Omega_{k+1}^{0}\right)+\frac{\xi}{h^{2}} \rho_{k}^{1}
\end{aligned}
$$

where $k=0: 1: N$. With the help of initial condition, $e^{0}=0$ :

$$
\begin{aligned}
\left(E_{1} b_{2}-\xi \Phi b_{5}\right) \Omega_{k}^{1}= & -\left(E_{1} b_{1}-\xi \Phi b_{4}\right)\left(\Omega_{k-1}^{1}+\Omega_{k+1}^{1}\right) \\
& +\xi \Psi b_{3}\left(\Omega_{k-1}^{1}-\Omega_{k+1}^{1}\right)+\frac{\xi}{h^{2}} \rho_{k}^{1} .
\end{aligned}
$$

Taking norms of $\Omega_{k}^{1}, \rho_{k}^{1}$ and adequately small mesh spacing $h$, we acquire

$e_{k}^{1} \leq \frac{3 \xi}{E_{1} h^{2}+12 \xi \Phi-3 \xi \Psi h} \rho h^{4}, \quad k=0: 1: N$.

We obtain the values of $e_{-1}^{1}$ and $e_{N+1}^{1}$ from the boundary conditions:

$e_{-1}^{1} \leq \frac{15 \xi}{E_{1} h^{2}+12 \xi \Phi-3 \xi \Psi h} \rho h^{4}$,

$e_{N+1}^{1} \leq \frac{15 \xi}{E_{1} h^{2}+12 \xi \Phi-3 \xi \Psi h} \rho h^{4}$,

which implies 
$e^{1} \leq \varrho_{1} h^{2}$,

where $\varrho_{1}$ is not depending on $h$.

To prove this theorem, mathematical induction on $m$ is used. For this, we consider that $e_{k}^{f} \leq \varrho_{f} h^{2}$ is true for $f=1,2, \ldots, m$ and $\rho=\max \left\{\varrho_{f}: f=0,1, \ldots, m\right\}$, then from Eq. (5.5), we obtain

$$
\begin{aligned}
& \left(E_{1} b_{1}-\xi \Phi b_{4}-\xi \Psi b_{3}\right) \Omega_{k-1}^{m+1} \\
& \quad+\left(E_{1} b_{2}-\xi \Phi b_{5}\right) \Omega_{k}^{m+1}+\left(E_{1} b_{1}-\xi \Phi b_{4}+\xi \Psi b_{3}\right) \Omega_{k+1}^{m+1} \\
& \quad=\left[\left(l_{0}-l_{1}\right)\left(b_{1} \Omega_{k-1}^{m}+b_{2} \Omega_{k}^{m}+b_{1} \Omega_{k+1}^{m}\right)\right. \\
& \quad+\left(l_{1}-l_{2}\right)\left(b_{1} \Omega_{k-1}^{m-1}+b_{2} \Omega_{k}^{m-1}+b_{1} \Omega_{k+1}^{m-1}\right)+\cdots \\
& \quad+\left(l_{m-1}-l_{m}\right)\left(b_{1} \Omega_{k-1}^{1}+b_{2} \Omega_{k}^{1}+b_{1} \Omega_{k+1}^{1}\right) \\
& \left.\quad+l_{m}\left(b_{1} \Omega_{k-1}^{0}+b_{2} \Omega_{k}^{0}+b_{1} \Omega_{k+1}^{0}\right)\right]+\frac{\xi}{h^{2}} \rho_{k}^{m+1} .
\end{aligned}
$$

Again, employing the norms on $\Omega_{k}^{m}$ and $\rho_{k}^{m}$, we acquire

$e_{k}^{m+1} \leq \frac{3 \rho h^{4}}{E_{1} h^{2}+12 \xi \Phi-3 \xi \Psi h}\left(\xi+\sum_{s=0}^{m-1}\left(l_{s}-l_{s+1}\right)\right)$.

Similarly, we get the values of $e_{-1}^{m+1}$ and $e_{N+1}^{m+1}$ from the boundary conditions:

$e_{-1}^{m+1} \leq \frac{15 \rho h^{4}}{E_{1} h^{2}+12 \xi \Phi-3 \xi \Psi h}\left(\xi+\sum_{s=0}^{m-1}\left(l_{s}-l_{s+1}\right)\right)$

$e_{N+1}^{m+1} \leq \frac{15 \rho h^{4}}{E_{1} h^{2}+12 \xi \Phi-3 \xi \Psi h}\left(\xi+\sum_{s=0}^{m-1}\left(l_{s}-l_{s+1}\right)\right)$.

Hence, for all $m$, we acquire

$e^{m+1} \leq \rho h^{2}$

In particular,

$\tilde{W}(r, t)-W(r, t)=\sum_{k=-1}^{N+1}\left(u_{k}(t)-\mu_{k}(t)\right) C_{k}(r)$.

Therefore, from Lemma 5.1 and inequality (5.7), we get

$\|\tilde{W}(r, t)-W(r, t)\|_{\infty} \leq \frac{5}{3} \rho h^{2}$.

Using (5.8), the inequality (5.4) gives

$\|w(r, t)-W(r, t)\|_{\infty} \leq \varrho_{0} h^{4}+\frac{5}{3} \rho h^{2}=\widetilde{\rho} h^{2}$,

where $\tilde{\varrho}=\varrho_{0} h^{2}+\frac{5}{3} \varrho$.

Theorem 4 The TFADE with initial and boundary conditions is convergent.
Proof Consider $w(r, t)$ and $W(r, t)$ be the solutions for TFADE analytically and numerically, respectively. Therefore, relation (3.3) and the above theorem validate that there are arbitrary constants $\widetilde{\varrho}$ and $\vartheta$, such that

$\|w(r, t)-W(r, t)\|_{\infty} \leq \widetilde{\rho} h^{2}+\vartheta(\Delta t)^{2}$.

Hence, the presented scheme is second order convergent in spatial and time directions.

\section{Illustration of numerical examples and discussion}

In this section, numerical outcomes are reported to check the accuracy of the scheme through the error norms $L_{2}(z), L_{\infty}(z)$ as

$$
\begin{aligned}
L_{2}(z) & =\left\|w\left(r_{k}, t\right)-W\left(r_{k}, t\right)\right\|_{2} \\
& =\sqrt{h \sum_{r=0}^{N}\left|w\left(r_{k}, t\right)-W\left(r_{k}, t\right)\right|^{2},} \\
L_{\infty}(z) & =\left\|w\left(r_{k}, t\right)-W\left(r_{k}, t\right)\right\|_{\infty} \\
& =\max _{0 \leq k \leq N}\left|w\left(r_{k}, t\right)-W\left(r_{k}, t\right)\right|
\end{aligned}
$$

and the convergence order [46] as

$\log \left(\frac{L_{\infty}(z)}{L_{\infty}(z+1)}\right) / \log \left(\frac{Q(z+1)}{Q(z)}\right)$.

All examples are solved by considering normalization function $R(\gamma)=1$.

Example 6.1 Consider the TFADE [46]

$$
\begin{aligned}
\frac{\partial^{\gamma} w(r, t)}{\partial t^{\gamma}}= & \frac{\partial^{2} w(r, t)}{\partial r^{2}}-\frac{\partial w(r, t)}{\partial r} \\
& +q(r, t), \quad r \in[0,1], t \in[0,1],
\end{aligned}
$$

with initial condition:

$w(r, 0)=0$

and boundary conditions:

$w(0, t)=0, w(1, t)=0$,

where $q(r, t)=2\left(\frac{R(\gamma)}{1-\gamma}\right) r(r-1) t^{2} E_{\gamma, 3}\left[\frac{-\gamma}{1-\gamma} \gamma^{\gamma}\right]-2 t^{2}+(2 r-1) t^{2}$.

The exact solution is $w(r, t)=r(r-1) t^{2}$. Tables 1 and 2 show the numerical outcomes and absolute errors of Example 6.1 for different choices of $\gamma$ setting $N=80,10,50$, $\Delta t=0.001$ and $t=1$. For different choices of $\gamma$, error norms are reported in Table 3 at various time stages. A Comparison of error norm and convergence order with those obtained in [46] is given in Tables 4 and 5 along temporal and spatial 


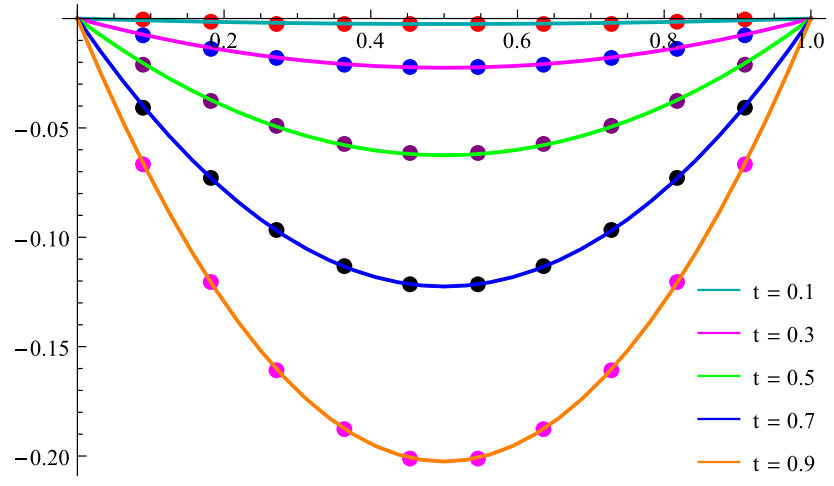

(a) $N=32, \gamma=0.1$

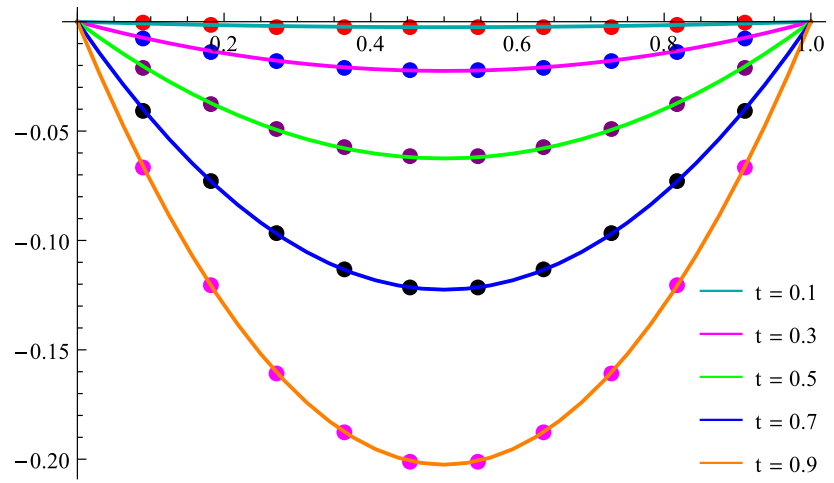

(c) $N=32, \gamma=0.5$

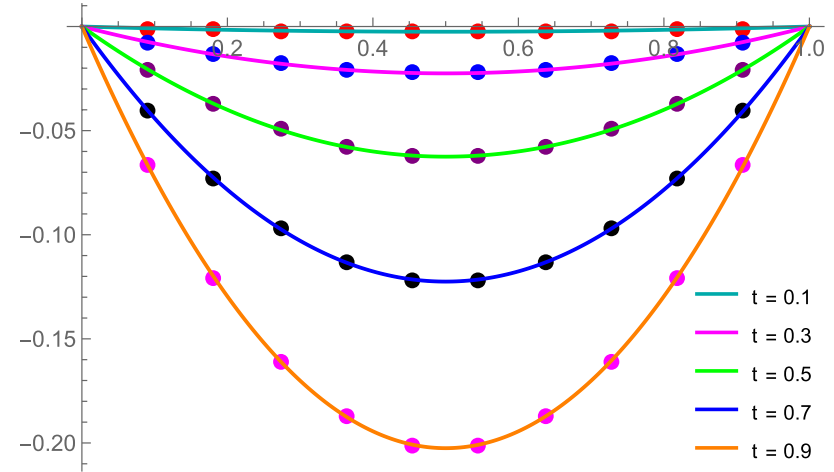

(e) $N=200, \gamma=0.9$

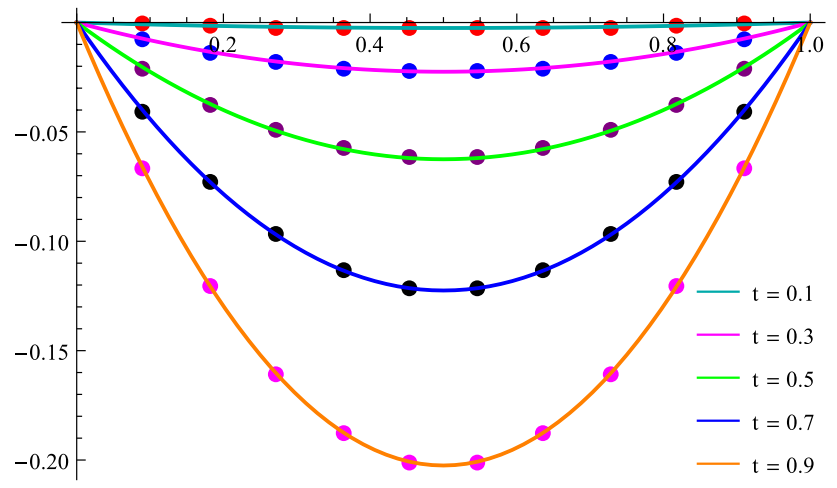

(b) $N=64, \gamma=0.1$

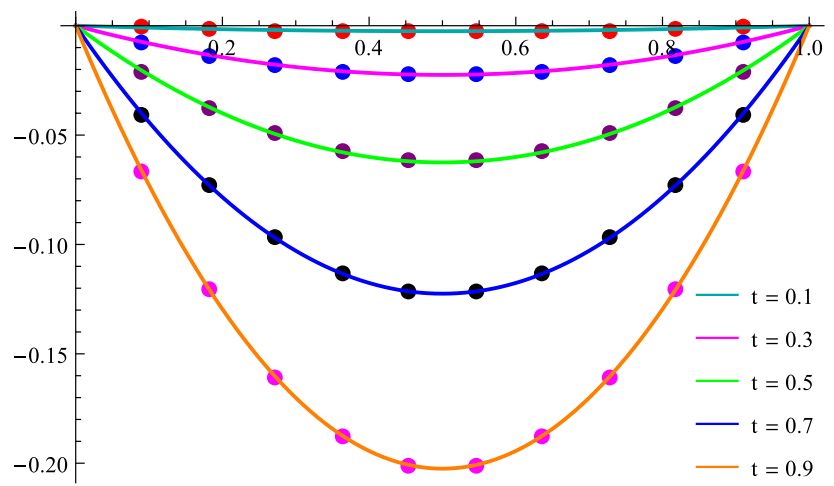

(d) $N=100, \gamma=0.5$

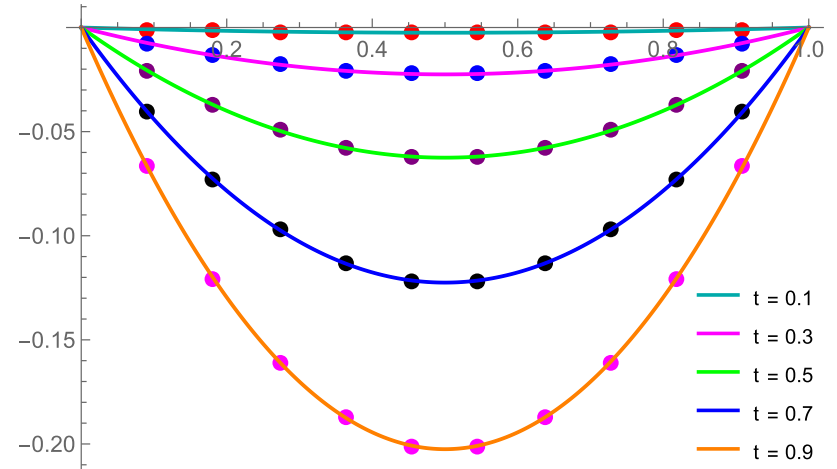

(f) $N=500, \gamma=0.9$

Fig. 1 Exact and numerical solutions for Example 6.1 at different time stages with $\Delta t=0.001$

directions. In Fig. 1, we can observe that close agreement between numerical results of the presented method and exact solutions at different time stages with $\Delta t=0.001$. A 3-D plot of exact solution and computational results is illustrated in Fig. 2 by setting $N=1000, \Delta t=0.001, \gamma=0.4, t=1$ and $r \in[0,1]$. In Fig. 3, the 2D and 3D error profiles are displayed at $t=1$. Tables and figures represent that the proposed method is compatible with stated exact solution.
Example 6.2 Consider the TFADE [46]

$$
\begin{aligned}
\frac{\partial^{\gamma} w(r, t)}{\partial t^{\gamma}}= & \frac{\partial^{2} w(r, t)}{\partial r^{2}}-\frac{\partial w(r, t)}{\partial r} \\
& +q(r, t), \quad r \in[0,1], t \in[0,1],
\end{aligned}
$$

with initial condition: 


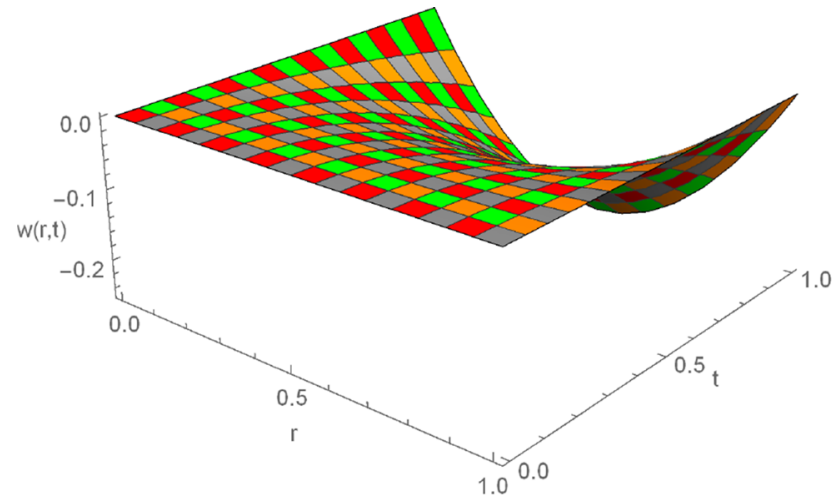

(a) Exact Solution

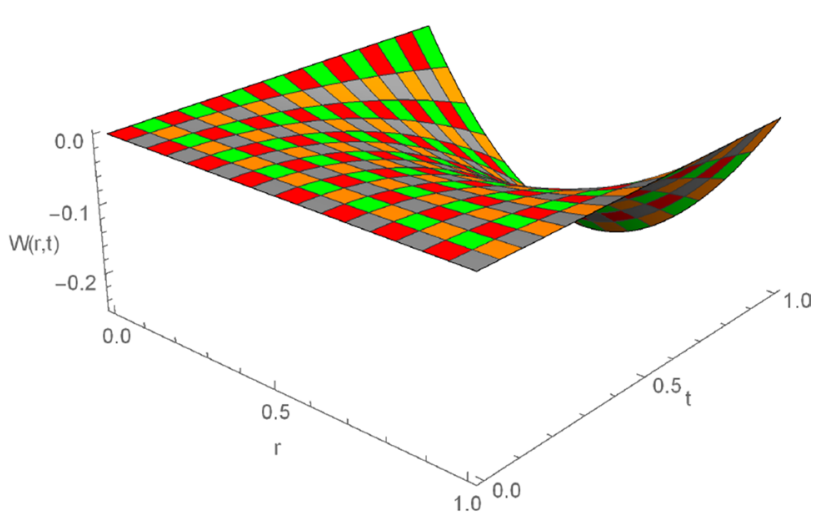

(b) Approximate Solution

Fig. 2 3D Exact and approximate solutions for Example 6.1, when $N=1000, t=1, \gamma=0.4, \Delta t=0.001$ and $r \in[0,1]$

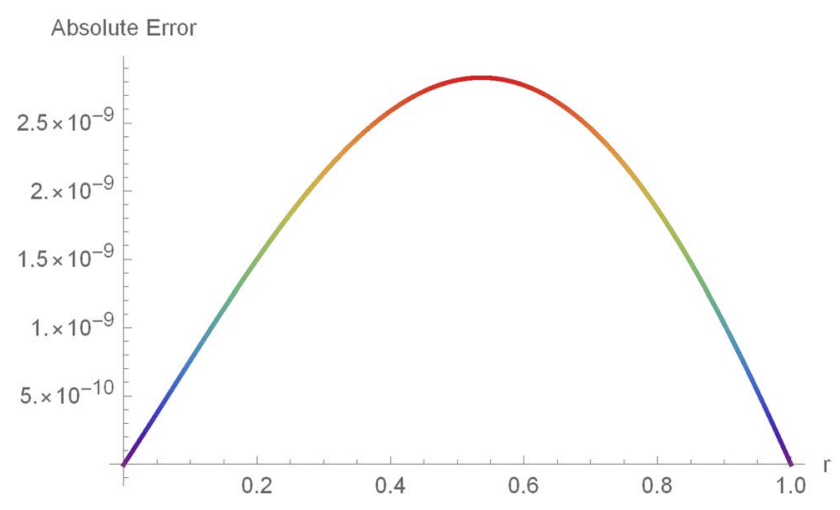

(a) 2D Error Function

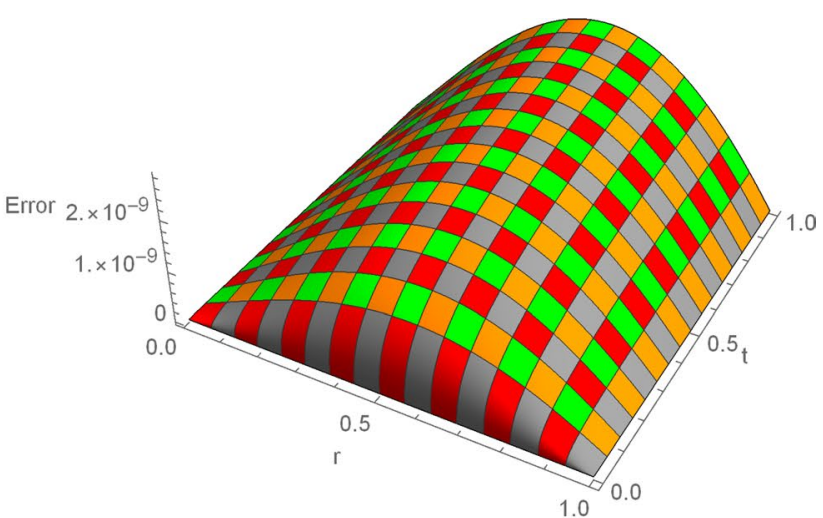

(b) 3D Error Function

Fig. $32 \mathrm{D}$ and 3D Error profiles for Example 6.1, when $N=1000, t=1, \gamma=0.4, \Delta t=0.001$ and $r \in[0,1]$

Table 1 Absolute errors for various choices of $\gamma$, where $\Delta t=0.001$ and $N=80$ of Example 6.1 at $t=1$

\begin{tabular}{lllllll}
\hline$r$ & Exact solution & \multicolumn{2}{l}{ Approximate solution } & \multicolumn{2}{l}{ Error } \\
\cline { 3 - 4 } \cline { 6 - 7 } & & $\gamma=0.2$ & $\gamma=0.7$ & $\gamma=0.2$ & $\gamma=0.7$ \\
\hline 0.1 & -0.09 & -0.09000000023560967 & -0.09000000289464635 & $2.35610 \times 10^{-10}$ & $2.89465 \times 10^{-9}$ \\
0.2 & -0.16 & -0.16000000046387822 & -0.16000000570048642 & & $4.63878 \times 10^{-10}$ & $5.70049 \times 10^{-9}$ \\
0.3 & -0.21 & -0.21000000065880656 & -0.21000000809766445 & & $6.58807 \times 10^{-10}$ & $8.09766 \times 10^{-9}$ \\
0.4 & -0.24 & -0.24000000079915515 & -0.2400000098246749 & & $7.99155 \times 10^{-10}$ & $9.82467 \times 10^{-9}$ \\
0.5 & -0.25 & -0.2500000008687607 & -0.2500000106821742 & & $8.68761 \times 10^{-10}$ & $1.06822 \times 10^{-8}$ \\
0.6 & -0.24 & -0.24000000085690387 & -0.2400000105377483 & & $8.56904 \times 10^{-10}$ & $1.05377 \times 10^{-8}$ \\
0.7 & -0.21 & -0.21000000075876923 & -0.21000000933177032 & $7.58769 \times 10^{-10}$ & $9.33177 \times 10^{-9}$ \\
0.8 & -0.16 & -0.16000000057601604 & -0.16000000708451972 & $5.76016 \times 10^{-10}$ & $7.08452 \times 10^{-9}$ \\
0.9 & -0.09 & -0.09000000031747607 & -0.09000000390475865 & $3.17476 \times 10^{-10}$ & $3.90476 \times 10^{-9}$ \\
\hline
\end{tabular}


Table 2 Absolute errors for Example 6.1 at $t=1$ and $\Delta t=0.001$

\begin{tabular}{lllllll}
\hline$r$ & Exact solution & \multicolumn{2}{l}{ Approximate solution } & & & \multicolumn{2}{l}{ Error } \\
\cline { 3 - 4 } & & $\gamma=0.1, N=10$ & $\gamma=0.7, N=50$ & & \multicolumn{2}{l}{$\gamma=0.1, N=10 \quad \gamma=0.7, N=50$} \\
\hline 0.1 & -0.09 & -0.09000000007298412 & -0.0900000028941835 & & $7.29841 \times 10^{-11}$ & $2.89418 \times 10^{-9}$ \\
0.2 & -0.16 & -0.16000000014375576 & -0.16000000569960401 & & $1.43756 \times 10^{-10}$ & $5.69960 \times 10^{-9}$ \\
0.3 & -0.21 & -0.2100000002042015 & -0.21000000809643382 & & $2.04202 \times 10^{-10}$ & $8.09643 \times 10^{-9}$ \\
0.4 & -0.24 & -0.2400000002477085 & -0.2400000098231799 & & $2.47709 \times 10^{-10}$ & $9.82318 \times 10^{-9}$ \\
0.5 & -0.25 & -0.25000000026925223 & -0.25000001068051464 & & $2.69252 \times 10^{-10}$ & $1.06805 \times 10^{-8}$ \\
0.6 & -0.24 & -0.24000000026551133 & -0.24000001053604475 & & $2.65511 \times 10^{-10}$ & $1.05360 \times 10^{-8}$ \\
0.7 & -0.21 & -0.21000000023501217 & -0.21000000933017113 & & $2.35012 \times 10^{-10}$ & $9.33017 \times 10^{-9}$ \\
0.8 & -0.16 & -0.1600000001783083 & -0.16000000708320752 & & $1.78308 \times 10^{-10}$ & $7.08321 \times 10^{-9}$ \\
0.9 & -0.09 & -0.09000000009819983 & -0.09000000390396098 & & $9.81998 \times 10^{-11}$ & $3.90396 \times 10^{-9}$ \\
\hline
\end{tabular}

Table 3 Error norms for various choices of $\gamma$, where $\Delta t=0.0005, N=32$ and $r \in[0,1]$ of Example 6.1

\begin{tabular}{|c|c|c|c|c|c|c|}
\hline \multirow[t]{2}{*}{$t$} & \multicolumn{3}{|l|}{$L_{\infty}(z)$} & \multicolumn{3}{|l|}{$L_{2}(z)$} \\
\hline & $\gamma=0.1$ & $\gamma=0.5$ & $\gamma=0.9$ & $\gamma=0.1$ & $\gamma=0.5$ & $\gamma=0.9$ \\
\hline 0.2 & $5.56352 \times 10^{-11}$ & $6.58804 \times 10^{-10}$ & $6.81249 \times 10^{-9}$ & $3.94425 \times 10^{-11}$ & $4.67133 \times 10^{-10}$ & $4.83419 \times 10^{-9}$ \\
\hline 0.4 & $6.20123 \times 10^{-11}$ & $8.40756 \times 10^{-10}$ & $8.82748 \times 10^{-9}$ & $4.39634 \times 10^{-11}$ & $5.96105 \times 10^{-10}$ & $6.25935 \times 10^{-9}$ \\
\hline 0.6 & $6.59160 \times 10^{-11}$ & $9.56088 \times 10^{-10}$ & $9.62124 \times 10^{-9}$ & $4.67308 \times 10^{-11}$ & $6.77845 \times 10^{-10}$ & $6.82017 \times 10^{-9}$ \\
\hline 0.8 & $6.87652 \times 10^{-11}$ & $1.04025 \times 10^{-9}$ & $1.03354 \times 10^{-8}$ & $4.87506 \times 10^{-11}$ & $7.37492 \times 10^{-10}$ & $7.32440 \times 10^{-9}$ \\
\hline 1.0 & $7.10227 \times 10^{-11}$ & $1.10611 \times 10^{-9}$ & $1.04419 \times 10^{-8}$ & $5.03511 \times 10^{-11}$ & $7.84161 \times 10^{-10}$ & $7.40045 \times 10^{-9}$ \\
\hline
\end{tabular}

Table 4 Comparison of error norm for Example 6.1 with different values of $\Delta t=\frac{1}{m}$ when $t=1$ and fixed $h=\frac{1}{1000}$

\begin{tabular}{|c|c|c|c|c|c|c|}
\hline \multirow[t]{2}{*}{$\gamma$} & \multirow[t]{2}{*}{$m$} & \multicolumn{2}{|l|}{ FDM [46] } & \multicolumn{3}{|l|}{ Proposed method } \\
\hline & & $L_{\infty}(z)$ & Order & $L_{\infty}(z)$ & $L_{2}(z)$ & Order \\
\hline \multirow[t]{5}{*}{0.2} & 10 & $6.29089 \times 10^{-6}$ & $\cdots$ & $6.29088 \times 10^{-6}$ & $4.45944 \times 10^{-6}$ & $\cdots$ \\
\hline & 20 & $1.69949 \times 10^{-6}$ & 1.888166062 & $1.69948 \times 10^{-6}$ & $1.20471 \times 10^{-6}$ & 1.888171257 \\
\hline & 40 & $4.53017 \times 10^{-7}$ & 1.907459519 & $4.53019 \times 10^{-7}$ & $3.21132 \times 10^{-7}$ & 1.907449346 \\
\hline & 80 & $1.19488 \times 10^{-7}$ & 1.922695207 & $1.19483 \times 10^{-7}$ & $8.46978 \times 10^{-8}$ & 1.922762688 \\
\hline & 160 & $3.12514 \times 10^{-8}$ & 1.934880530 & $3.12511 \times 10^{-8}$ & $2.21536 \times 10^{-8}$ & 1.934828974 \\
\hline \multirow[t]{5}{*}{0.3} & 10 & $1.34627 \times 10^{-5}$ & $\cdots$ & $1.34627 \times 10^{-5}$ & $9.54335 \times 10^{-6}$ & $\cdots$ \\
\hline & 20 & $3.58496 \times 10^{-6}$ & 1.908944525 & $3.58495 \times 10^{-6}$ & $2.54127 \times 10^{-6}$ & 1.908943810 \\
\hline & 40 & $9.41934 \times 10^{-7}$ & 1.928258301 & $9.41931 \times 10^{-7}$ & $6.67707 \times 10^{-7}$ & 1.928261075 \\
\hline & 80 & $2.44961 \times 10^{-7}$ & 1.943071838 & $2.44959 \times 10^{-7}$ & $1.73645 \times 10^{-7}$ & 1.943079673 \\
\hline & 160 & $6.31993 \times 10^{-8}$ & 1.954573069 & $6.31974 \times 10^{-8}$ & $4.47988 \times 10^{-8}$ & 1.954605365 \\
\hline \multirow[t]{5}{*}{0.4} & 10 & $2.38602 \times 10^{-5}$ & $\cdots$ & $2.38601 \times 10^{-5}$ & $1.69138 \times 10^{-5}$ & $\cdots$ \\
\hline & 20 & $6.27827 \times 10^{-6}$ & 1.926164892 & $6.27826 \times 10^{-6}$ & $4.45047 \times 10^{-6}$ & 1.926163821 \\
\hline & 40 & $1.63031 \times 10^{-6}$ & 1.945222205 & $1.630298 \times 10^{-6}$ & $1.15567 \times 10^{-6}$ & 1.945230090 \\
\hline & 80 & $4.19281 \times 10^{-7}$ & 1.959154613 & $4.19280 \times 10^{-7}$ & $2.97214 \times 10^{-7}$ & 1.959148810 \\
\hline & 160 & $1.07066 \times 10^{-7}$ & 1.969412862 & $1.07068 \times 10^{-7}$ & $7.58968 \times 10^{-8}$ & 1.969391952 \\
\hline \multirow[t]{5}{*}{0.5} & 10 & $3.90135 \times 10^{-5}$ & $\cdots$ & $3.90134 \times 10^{-5}$ & $2.76554 \times 10^{-5}$ & $\cdots$ \\
\hline & 20 & $1.01634 \times 10^{-5}$ & 1.940583396 & $1.01634 \times 10^{-5}$ & $7.20451 \times 10^{-6}$ & 1.940583037 \\
\hline & 40 & $2.61414 \times 10^{-6}$ & 1.958980182 & $2.61414 \times 10^{-6}$ & $1.85307 \times 10^{-6}$ & 1.958977081 \\
\hline & 80 & $6.66564 \times 10^{-7}$ & 1.971522766 & $6.66564 \times 10^{-7}$ & $4.72503 \times 10^{-7}$ & 1.971524280 \\
\hline & 160 & $1.68953 \times 10^{-7}$ & 1.980125119 & $1.68954 \times 10^{-7}$ & $1.19766 \times 10^{-7}$ & 1.980113110 \\
\hline
\end{tabular}


Table 5 Comparison of error norm for Example 6.1 with different values of $\Delta t=\frac{1}{m}$ and $h=\frac{1}{n}$ when $t=1$

\begin{tabular}{|c|c|c|c|c|c|c|}
\hline \multirow[t]{2}{*}{$\gamma$} & \multirow[t]{2}{*}{$m=n$} & \multicolumn{2}{|l|}{ FDM [46] } & \multicolumn{3}{|l|}{ Proposed method } \\
\hline & & $L_{\infty}(z)$ & Order & $L_{\infty}(z)$ & $L_{2}(z)$ & Order \\
\hline \multirow[t]{5}{*}{0.2} & 10 & $6.30203 \times 10^{-6}$ & $\cdots$ & $6.21118 \times 10^{-6}$ & $4.42953 \times 10^{-6}$ & $\cdots$ \\
\hline & 20 & $1.70133 \times 10^{-6}$ & 1.889155874 & $1.69517 \times 10^{-6}$ & $1.2027 \times 10^{-6}$ & 1.873440564 \\
\hline & 40 & $4.52969 \times 10^{-7}$ & 1.909176733 & $4.52558 \times 10^{-7}$ & $3.20997 \times 10^{-7}$ & 1.905251532 \\
\hline & 80 & $1.19502 \times 10^{-7}$ & 1.922377832 & $1.19475 \times 10^{-7}$ & $8.46927 \times 10^{-8}$ & 1.921396468 \\
\hline & 160 & $3.12501 \times 10^{-8}$ & 1.935099332 & $3.12486 \times 10^{-8}$ & $2.21514 \times 10^{-8}$ & 1.934844164 \\
\hline \multirow[t]{5}{*}{0.3} & 10 & $1.34866 \times 10^{-5}$ & $\cdots$ & $1.32921 \times 10^{-5}$ & $9.47935 \times 10^{-6}$ & $\cdots$ \\
\hline & 20 & $3.58884 \times 10^{-6}$ & 1.909933591 & $3.57584 \times 10^{-6}$ & $2.53702 \times 10^{-6}$ & 1.894216888 \\
\hline & 40 & $9.41832 \times 10^{-7}$ & 1.929977781 & $9.40978 \times 10^{-7}$ & $6.6743 \times 10^{-7}$ & 1.926051621 \\
\hline & 80 & $2.44988 \times 10^{-7}$ & 1.942760823 & $2.44932 \times 10^{-7}$ & $1.73626 \times 10^{-7}$ & 1.941778797 \\
\hline & 160 & $6.31982 \times 10^{-8}$ & 1.954754361 & $6.31947 \times 10^{-8}$ & $4.47971 \times 10^{-8}$ & 1.954507116 \\
\hline \multirow[t]{5}{*}{0.4} & 10 & $2.39024 \times 10^{-5}$ & $\cdots$ & $2.35577 \times 10^{-5}$ & $1.68003 \times 10^{-5}$ & $\cdots$ \\
\hline & 20 & $6.28509 \times 10^{-6}$ & 1.927152757 & $6.26231 \times 10^{-6}$ & $4.44303 \times 10^{-6}$ & 1.911433379 \\
\hline & 40 & $1.63013 \times 10^{-6}$ & 1.946946105 & $1.62865 \times 10^{-6}$ & $1.15519 \times 10^{-6}$ & 1.943018626 \\
\hline & 80 & $4.19330 \times 10^{-7}$ & 1.958828865 & $4.19235 \times 10^{-7}$ & $2.97183 \times 10^{-7}$ & 1.957846640 \\
\hline & 160 & $1.07068 \times 10^{-7}$ & 1.969561794 & $1.07062 \times 10^{-7}$ & $7.58931 \times 10^{-8}$ & 1.969316007 \\
\hline \multirow[t]{5}{*}{0.5} & 10 & $3.90827 \times 10^{-5}$ & $\cdots$ & $3.85187 \times 10^{-5}$ & $2.74697 \times 10^{-5}$ & $\cdots$ \\
\hline & 20 & $1.01745 \times 10^{-5}$ & 1.941570692 & $1.01376 \times 10^{-5}$ & $7.19245 \times 10^{-6}$ & 1.925839438 \\
\hline & 40 & $2.61386 \times 10^{-6}$ & 1.960707916 & $2.61148 \times 10^{-6}$ & $1.8523 \times 10^{-6}$ & 1.956776747 \\
\hline & 80 & $6.66644 \times 10^{-7}$ & 1.971192408 & $6.66492 \times 10^{-7}$ & $4.72454 \times 10^{-7}$ & 1.970209326 \\
\hline & 160 & $1.68955 \times 10^{-7}$ & 1.980273364 & $1.68946 \times 10^{-7}$ & $1.1976 \times 10^{-7}$ & 1.980028239 \\
\hline
\end{tabular}

Table 6 Absolute error for Example 6.2 at $t=1, \gamma=0.2$, $\Delta t=0.0125$ and $N=500$

Table 7 Absolute error for Example 6.2 at $t=1, \gamma=0.4$, $\Delta t=0.05$ and $N=70$

\begin{tabular}{llll}
\hline$r$ & Exact solution & Approximate solution & Error \\
\hline 0.1 & 0.30901699437494745 & 0.3090141613478385 & $2.8330271089616055 \times 10^{-6}$ \\
0.2 & 0.5877852522924731 & 0.587779638352816 & $5.613939657145295 \times 10^{-6}$ \\
0.3 & 0.8090169943749475 & 0.8090089728017736 & $8.021573173810914 \times 10^{-6}$ \\
0.4 & 0.9510565162951535 & 0.9510467467983879 & $9.769496765676955 \times 10^{-6}$ \\
0.5 & 1 & 0.999989367097764 & $1.0632902236018538 \times 10^{-5}$ \\
0.6 & 0.9510565162951535 & 0.9510460470148395 & $1.0469280314007179 \times 10^{-5}$ \\
0.7 & 0.8090169943749475 & 0.8090077635576107 & $9.230817336725039 \times 10^{-6}$ \\
0.8 & 0.5877852522924731 & 0.5877782850448111 & $6.967247662026388 \times 10^{-6}$ \\
0.9 & 0.30901699437494745 & 0.30901317555384294 & $3.818821104506487 \times 10^{-6}$
\end{tabular}

\begin{tabular}{llll}
\hline$r$ & Exact solution & Approximate solution & Error \\
\hline 0.1 & 0.30901699437494745 & 0.30901702338700715 & $2.9012059699073944 \times 10^{-8}$ \\
0.2 & 0.5877852522924731 & 0.5877853098089763 & $5.751650311580647 \times 10^{-8}$ \\
0.3 & 0.8090169943749475 & 0.8090170766181584 & $8.224321090821718 \times 10^{-8}$ \\
0.4 & 0.9510565162951535 & 0.9510566165465532 & $1.0025139962621665 \times 10^{-7}$ \\
0.5 & 1 & 1.00000010921341 & $1.0921341009328955 \times 10^{-7}$ \\
0.6 & 0.9510565162951535 & 0.9510566239287926 & $1.0763363911259205 \times 10^{-7}$ \\
0.7 & 0.8090169943749475 & 0.8090170893593546 & $9.498440711741551 \times 10^{-8}$ \\
0.8 & 0.5877852522924731 & 0.5877853240383313 & $7.174585814340162 \times 10^{-8}$ \\
0.9 & 0.30901699437494745 & 0.3090170337198961 & $3.9344948632447085 \times 10^{-8}$ \\
\hline
\end{tabular}


Table 8 Error norms for various choices of $\gamma$, where $\Delta t=0.01$, $N=85$ and $r \in[0,1]$ of Example 6.2

\begin{tabular}{|c|c|c|c|c|c|c|}
\hline \multirow[t]{2}{*}{$t$} & \multicolumn{3}{|l|}{$L_{\infty}(z)$} & \multicolumn{3}{|l|}{$L_{2}(z)$} \\
\hline & $\gamma=0.2$ & $\gamma=0.5$ & $\gamma=0.8$ & $\gamma=0.2$ & $\gamma=0.5$ & $\gamma=0.8$ \\
\hline 0.2 & $2.24118 \times 10^{-8}$ & $7.09333 \times 10^{-9}$ & $1.06536 \times 10^{-7}$ & $1.5824 \times 10^{-8}$ & $5.00854 \times 10^{-9}$ & $7.52367 \times 10^{-8}$ \\
\hline 0.4 & $9.37425 \times 10^{-7}$ & $5.75672 \times 10^{-7}$ & $7.75183 \times 10^{-7}$ & $6.61875 \times 10^{-7}$ & $4.06481 \times 10^{-7}$ & $5.47406 \times 10^{-7}$ \\
\hline 0.6 & $7.50231 \times 10^{-6}$ & $5.91274 \times 10^{-6}$ & $1.75069 \times 10^{-7}$ & $5.29705 \times 10^{-6}$ & $4.17495 \times 10^{-6}$ & $1.23401 \times 10^{-7}$ \\
\hline 0.8 & $3.22589 \times 10^{-5}$ & $2.76668 \times 10^{-5}$ & $1.01196 \times 10^{-5}$ & $2.27765 \times 10^{-5}$ & $1.95352 \times 10^{-5}$ & $7.14637 \times 10^{-6}$ \\
\hline 1.0 & $9.94523 \times 10^{-5}$ & $8.89304 \times 10^{-5}$ & $4.92972 \times 10^{-5}$ & $7.02186 \times 10^{-5}$ & $6.27927 \times 10^{-5}$ & $3.48118 \times 10^{-5}$ \\
\hline
\end{tabular}

\begin{tabular}{llllll}
\hline$t$ & \multicolumn{2}{l}{$L_{\infty}(z)$} & & \multicolumn{2}{l}{$L_{2}(z)$} \\
\cline { 2 - 3 } \cline { 5 - 6 } & $\gamma=0.1$ & $\gamma=0.7$ & & $\gamma=0.1$ & $\gamma=0.7$ \\
\hline 0.2 & $4.29501 \times 10^{-10}$ & $7.56327 \times 10^{-8}$ & & $3.03202 \times 10^{-10}$ & $5.34027 \times 10^{-8}$ \\
0.4 & $6.05272 \times 10^{-8}$ & $8.49359 \times 10^{-7}$ & & $4.27287 \times 10^{-8}$ & $5.99692 \times 10^{-7}$ \\
0.6 & $5.84265 \times 10^{-7}$ & $3.24254 \times 10^{-6}$ & & $4.12457 \times 10^{-7}$ & $2.28934 \times 10^{-6}$ \\
0.8 & $2.66452 \times 10^{-6}$ & $7.83032 \times 10^{-6}$ & & $1.88100 \times 10^{-6}$ & $5.52835 \times 10^{-6}$ \\
1.0 & $8.43719 \times 10^{-6}$ & $1.43745 \times 10^{-5}$ & & $5.95616 \times 10^{-6}$ & $1.01484 \times 10^{-5}$ \\
\hline
\end{tabular}

Table 9 Error norms for Example 6.2 when $\Delta t=0.01$, $N=285$ and $r \in[0,1]$ $w(r, 0)=0$

and boundary conditions:

$w(0, t)=0, \quad w(1, t)=0$,

where $q(r, t)=120\left(\frac{R(\gamma)}{1-\gamma}\right) t^{5} \sin (\pi r) E_{\gamma, 6}\left[\frac{-\gamma}{1-\gamma} t^{\gamma}\right]+\pi t^{5}(\pi \sin$ $(\pi r)+\cos (\pi r))$

The exact solution is $w(r, t)=t^{5} \sin (\pi r)$. The absolute errors for Example 6.2 at numerous values of $r$ setting $\Delta t=0.0125,0.05, N=500,70, \gamma=0.2,0.4$ and $t=1$ are tabulated in Tables 6 and 7. Tables 8 and 9 display the error norms for different values of $\gamma$ subject to $N=85,285$, $\Delta t=0.01$ and $r \in[0,1]$ at various time stages. Comparison of convergence order and errors are displayed in Tables 10 and 11 in temporal and spatial grids, respectively. It is found that the proposed scheme shows better efficiency and accuracy as compared to the scheme proposed in [46]. Figure 4 illustrates the behavior of computational results and exact values at different time stages. In Fig. 5, 3-D graphs of numerical outcomes and analytical solutions depict the accuracy of current scheme. Figure 6 shows the 2D and 3D error profiles, which exhibit exactness of the method.

\section{Conclusion}

In current paper, an efficient solution of TFADE involving Atangana-Baleanu time derivative has attained through numerical scheme based on CBS functions. The standard finite difference formulation has been used to approximate the Atangana-Baleanu time fractional derivative, while interpolation of solution curve in spatial direction is obtained through CBS functions. The method proposed in current study is novel and provides reasonable accuracy when compared to those already developed in literature. The method is unconditionally stable along with second order spatial and temporal convergence. The implementation of the current algorithm on numerical examples reveal this method to be more efficient, simple and admissible. 
Table 10 Comparison of error norm for Example 6.2 with different values of $\Delta t=\frac{1}{m}$ when $t=1$ and fixed $h=\frac{1}{1000}$

\begin{tabular}{|c|c|c|c|c|c|c|}
\hline \multirow[t]{2}{*}{$\gamma$} & \multirow[t]{2}{*}{$m$} & \multicolumn{2}{|l|}{ FDM [46] } & \multicolumn{3}{|c|}{ Proposed method } \\
\hline & & $L_{\infty}(z)$ & Order & $L_{\infty}(z)$ & $L_{2}(z)$ & Order \\
\hline \multirow[t]{5}{*}{0.2} & 10 & 0.000139981 & $\cdots$ & 0.000138503 & $9.7775 \times 10^{-5}$ & $\cdots$ \\
\hline & 20 & $4.08956 \times 10^{-5}$ & 1.775214940 & $3.9418 \times 10^{-5}$ & $2.78267 \times 10^{-5}$ & 1.812994737 \\
\hline & 40 & $1.19251 \times 10^{-5}$ & 1.777941708 & $1.04467 \times 10^{-5}$ & $7.37474 \times 10^{-6}$ & 1.915806402 \\
\hline & 80 & $3.79060 \times 10^{-6}$ & 1.653504636 & $2.30918 \times 10^{-6}$ & $1.63014 \times 10^{-6}$ & 2.177596014 \\
\hline & 160 & $1.56917 \times 10^{-6}$ & 1.272422075 & $8.14794 \times 10^{-8}$ & $5.75207 \times 10^{-8}$ & 4.824801237 \\
\hline \multirow[t]{5}{*}{0.3} & 10 & 0.000287302 & $\cdots$ & 0.000285834 & 0.000201783 & $\cdots$ \\
\hline & 20 & $8.16643 \times 10^{-5}$ & 1.814789261 & $8.01969 \times 10^{-5}$ & $5.66146 \times 10^{-5}$ & 1.833557126 \\
\hline & 40 & $2.28252 \times 10^{-5}$ & 1.839076189 & $2.13575 \times 10^{-5}$ & $1.50772 \times 10^{-5}$ & 1.908801582 \\
\hline & 80 & $6.63756 \times 10^{-6}$ & 1.781904211 & $5.16816 \times 10^{-6}$ & $3.64844 \times 10^{-6}$ & 2.047022699 \\
\hline & 160 & $2.29644 \times 10^{-6}$ & 1.531255342 & $8.22282 \times 10^{-7}$ & $5.80484 \times 10^{-7}$ & 2.651945595 \\
\hline \multirow[t]{5}{*}{0.4} & 10 & 0.000493326 & $\cdots$ & 0.000491872 & 0.000347239 & $\cdots$ \\
\hline & 20 & 0.000137198 & 1.846284896 & 0.000135745 & $9.58295 \times 10^{-5}$ & 1.857385833 \\
\hline & 40 & $3.73104 \times 10^{-5}$ & 1.878605287 & $3.58575 \times 10^{-5}$ & $2.53137 \times 10^{-5}$ & 1.920548663 \\
\hline & 80 & $1.03383 \times 10^{-5}$ & 1.851583641 & $8.88435 \times 10^{-6}$ & $6.27193 \times 10^{-6}$ & 2.012938781 \\
\hline & 160 & $3.22472 \times 10^{-6}$ & 1.680750781 & $1.76728 \times 10^{-6}$ & $1.24761 \times 10^{-6}$ & 2.329737231 \\
\hline \multirow[t]{5}{*}{0.5} & 10 & 0.00078925 & $\cdots$ & 0.000787815 & 0.00055617 & $\cdots$ \\
\hline & 20 & 0.00021535 & 1.873801387 & 0.000213917 & 0.000151018 & 1.880808105 \\
\hline & 40 & $5.73409 \times 10^{-5}$ & 1.909044348 & $5.59084 \times 10^{-5}$ & $3.94693 \times 10^{-5}$ & 1.935913970 \\
\hline & 80 & $1.53859 \times 10^{-5}$ & 1.897956775 & $1.39527 \times 10^{-5}$ & $9.8501 \times 10^{-6}$ & 2.002522561 \\
\hline & 160 & $4.47913 \times 10^{-6}$ & 1.780318285 & $3.04332 \times 10^{-6}$ & $2.14848 \times 10^{-6}$ & 2.196821079 \\
\hline
\end{tabular}

Table 11 Comparison of error norm for Example 6.2 with different values of $h=\frac{1}{n}$ and $\Delta t=\frac{1}{500}$ when $t=1$

\begin{tabular}{|c|c|c|c|c|c|c|}
\hline \multirow[t]{2}{*}{$\gamma$} & \multirow[t]{2}{*}{$n$} & \multicolumn{2}{|l|}{ FDM [46] } & \multicolumn{3}{|l|}{ Proposed method } \\
\hline & & $L_{\infty}(z)$ & Order & $L_{\infty}(z)$ & $L_{2}(z)$ & Order \\
\hline \multirow[t]{5}{*}{0.2} & 10 & 0.007622189 & $\cdots$ & 0.00726005 & 0.0051572 & $\cdots$ \\
\hline & 20 & 0.001915395 & 1.992563186 & 0.00182915 & 0.00129262 & 1.988806934 \\
\hline & 40 & 0.000478550 & 2.000901471 & 0.000457745 & 0.000323317 & 1.998557412 \\
\hline & 80 & 0.000119693 & 1.999324187 & 0.000114447 & 0.000080794 & 1.999869299 \\
\hline & 160 & $2.99395 \times 10^{-5}$ & 1.999223018 & $2.85445 \times 10^{-5}$ & $2.01511 \times 10^{-5}$ & 2.003389455 \\
\hline \multirow[t]{5}{*}{0.3} & 10 & 0.007569951 & $\cdots$ & 0.00721124 & 0.00512245 & $\cdots$ \\
\hline & 20 & 0.001902765 & 1.992186298 & 0.00181667 & 0.00128383 & 1.988951007 \\
\hline & 40 & 0.000475413 & 2.000842977 & 0.000454567 & 0.000321073 & 1.998729270 \\
\hline & 80 & 0.000118918 & 1.999219732 & 0.000113592 & $8.01918 \times 10^{-5}$ & 2.000630037 \\
\hline & 160 & $2.97599 \times 10^{-5}$ & 1.998523576 & $2.82727 \times 10^{-5}$ & $1.99595 \times 10^{-5}$ & 2.006381843 \\
\hline \multirow[t]{5}{*}{0.4} & 10 & 0.007494668 & $\cdots$ & 0.00714089 & 0.00507238 & $\cdots$ \\
\hline & 20 & 0.001884556 & 1.991639999 & 0.001798697 & 0.00127117 & 1.989151495 \\
\hline & 40 & 0.000470889 & 2.000766894 & 0.000450004 & 0.000317851 & 1.998943997 \\
\hline & 80 & 0.000117795 & 1.999103804 & 0.000112376 & $7.93348 \times 10^{-5}$ & 2.001597147 \\
\hline & 160 & $2.94972 \times 10^{-5}$ & 1.997633680 & $2.78965 \times 10^{-5}$ & $1.96942 \times 10^{-5}$ & 2.010185304 \\
\hline \multirow[t]{5}{*}{0.5} & 10 & 0.007390490 & $\cdots$ & 0.00704352 & 0.00500306 & $\cdots$ \\
\hline & 20 & 0.001859347 & 1.990873841 & 0.00177383 & 0.00125365 & 1.989430161 \\
\hline & 40 & 0.000464623 & 2.000662501 & 0.000443691 & 0.000313394 & 1.999237222 \\
\hline & 80 & 0.000116240 & 1.998949703 & 0.000110698 & $7.81519 \times 10^{-5}$ & 2.002925008 \\
\hline & 160 & $2.91333 \times 10^{-5}$ & 1.996370865 & $2.73802 \times 10^{-5}$ & $1.93302 \times 10^{-5}$ & 2.015425048 \\
\hline
\end{tabular}




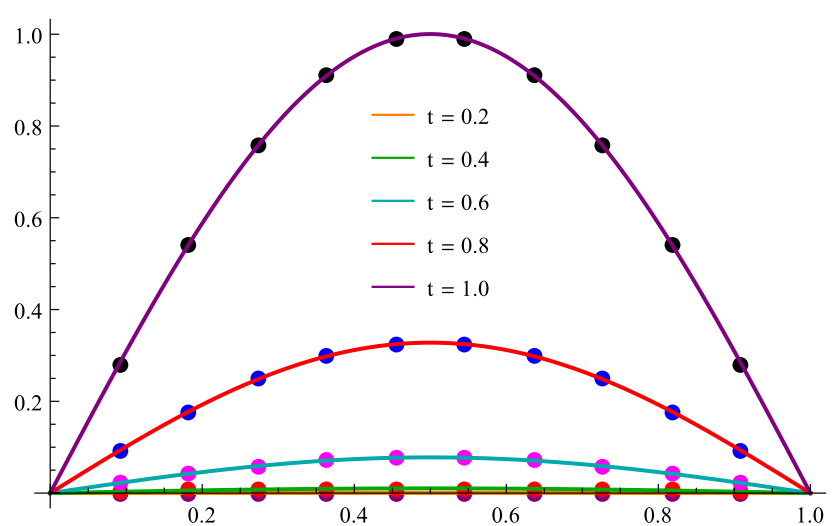

(a) $N=160, \gamma=0.9$ and $\Delta t=0.02$

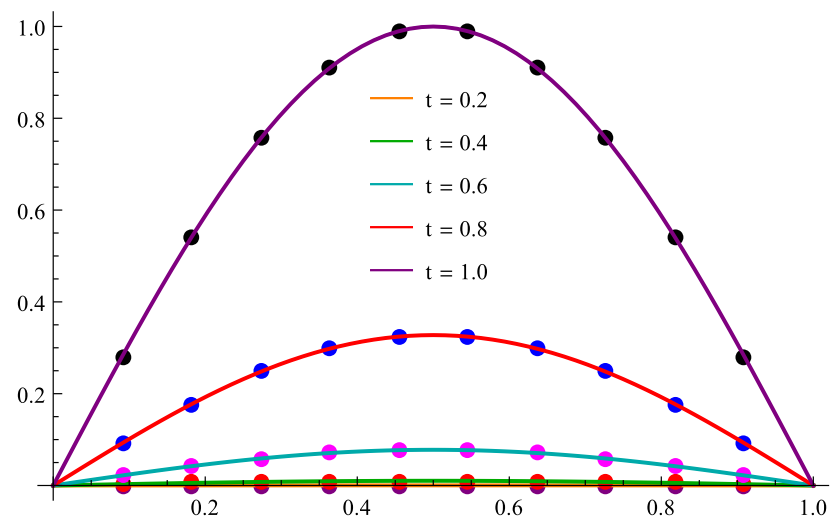

(c) $N=64, \gamma=0.2$ and $\Delta t=0.002$

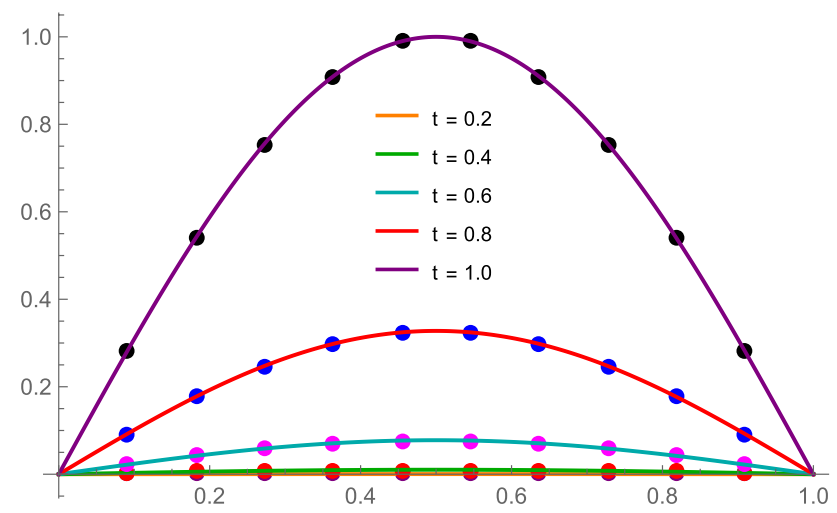

(e) $N=200, \gamma=0.4$ and $\Delta t=0.0025$

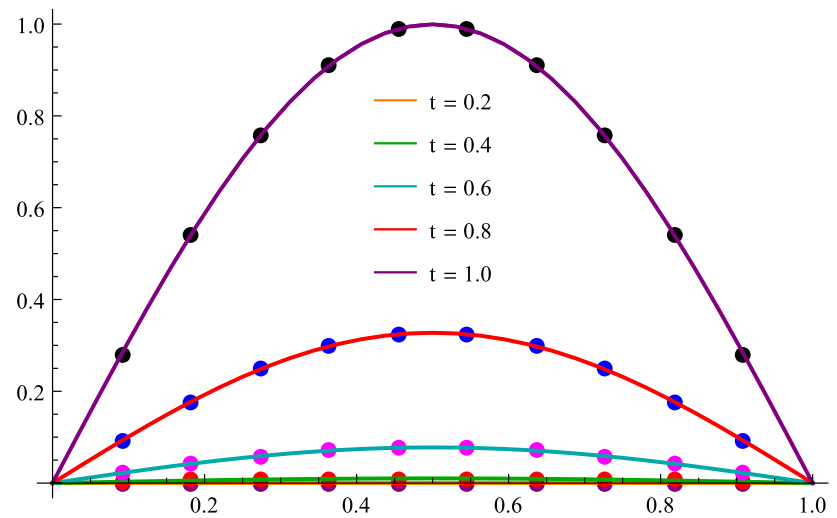

(b) $N=32, \gamma=0.7$ and $\Delta t=0.01$

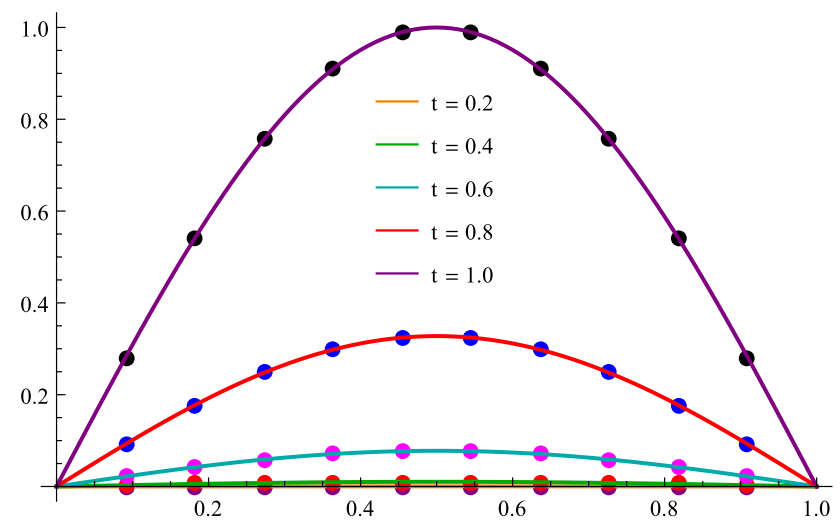

(d) $N=100, \gamma=0.5$ and $\Delta t=0.001$

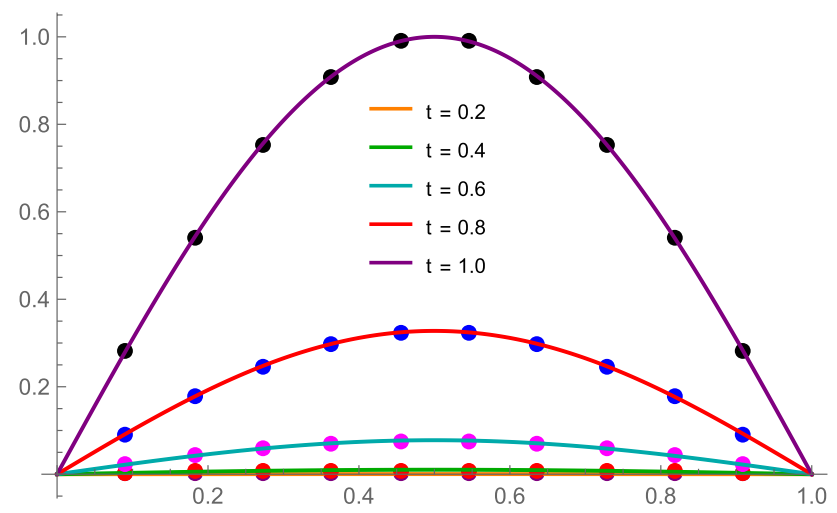

(f) $N=500, \gamma=0.6$ and $\Delta t=0.005$

Fig. 4 Exact and numerical solutions for Example 6.2 at different time stages 


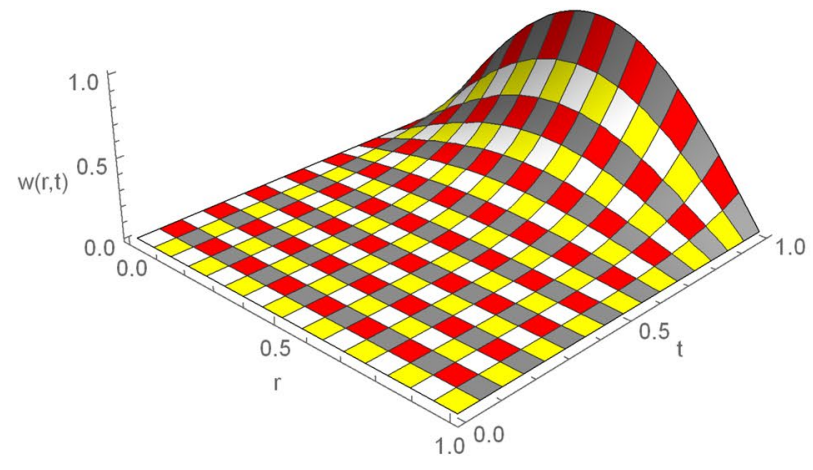

(a) Exact Solution

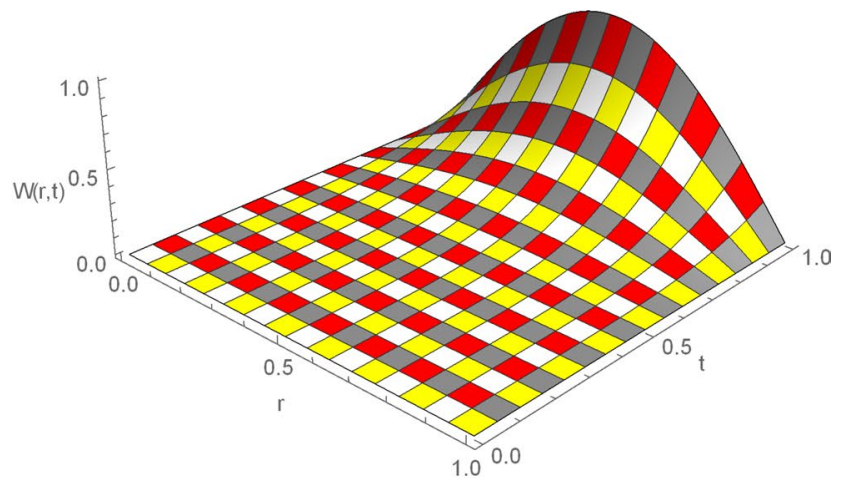

(b) Approximate Solution

Fig. $53 \mathrm{D}$ exact and approximate solutions for Example 6.2, when $N=1000, t=1, \gamma=0.4, \Delta t=0.001$ and $r \in[0,1]$

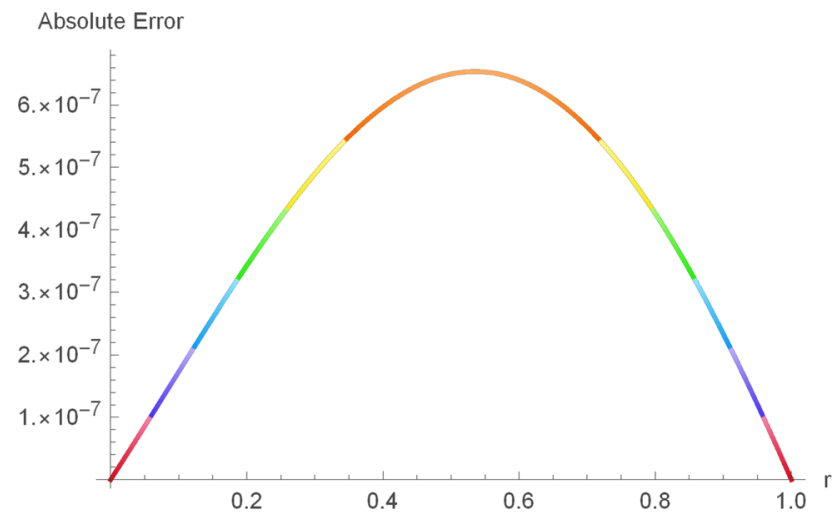

(a) 2D Error Function

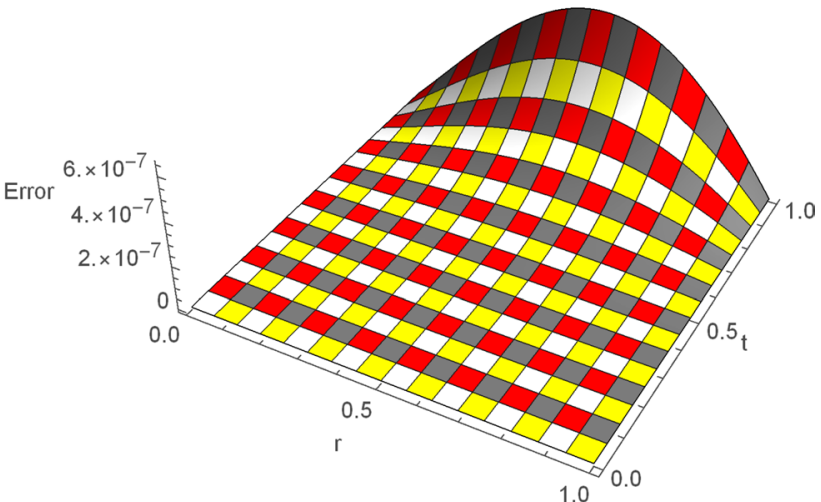

(b) 3D Error Function

Fig. $62 \mathrm{D}$ and 3D error profiles for Example 6.2, when $N=1000, t=1, \gamma=0.4, \Delta t=0.001$ and $r \in[0,1]$

Acknowledgements This Research was supported by Taif University Researchers Supporting Project Number (TURSP-2020/217), Taif University, Taif, Saudi Arabia. We thank Dr. Muhammad Kashif Iqbal for his assistance in proofreading of the manuscript. The authors are also grateful to anonymous referees for their valuable suggestions, which significantly improved this manuscript.

Author Contributions All authors equally contributed to this work. All authors read and approved the final manuscript.

\section{Declarations}

Competing interests The authors declare that they have no conflicts of interest to report regarding the present study.

\section{References}

1. Diethelm K, Freed AD (1999) On the solution of nonlinear fractional-order differential equations used in the modeling of viscoplasticity. In: Scientific computing in chemical engineering II. Springer, Berlin, Heidelberg, pp 217-224

2. Sokolov IM, Klafter J, Blumen A (2002) Fractional kinetics. Phys Today 55(11):48-54

3. Hilfer R (ed) (2000) Applications of fractional calculus in physics, vol 35. World Scientific, Singapore

4. Bokhari AH, Kara AH, Zaman FD (2009) On the solutions and conservation laws of the model for tumor growth in the brain. $\mathrm{J}$ Math Anal Appl 350(1):256-261

5. Mainardi F (1997) Fractional calculus. In: Fractals and fractional calculus in continuum mechanics. Springer, Vienna, pp 291-348

6. Metzler R, Klafter J (2000) The random walks guide to anomalous diffusion: a fractional dynamics approach. Phys Rep 339(1):1-77

7. Sokolov IM, Klafter J, Blumen A (2000) Ballistic versus diffusive pair dispersion in the Richardson regime. Phys Rev E 61(3):2717-2722

8. Chen W (2006) A speculative study of 2/3-order fractional laplacian modeling of turbulence: some thoughts and conjectures. Chaos Interdiscip J Nonlinear Sci 16(2):023126

9. Atangana A, Baleanu D (2016) New fractional derivatives with nonlocal and non-singular kernel: theory and application to heat transfer model. Therm Sci 20(2):763-769 
10. Alkahtani BST (2016) Chuas circuit model with atangana-baleanu derivative with fractional order. Chaos Solitons Fract 89:547-551

11. Gómez-Aguilar JF (2017) Irving-mullineux oscillator via fractional derivatives with mittag-leffler kernel. Chaos Solitons Fract 95:179-186

12. Prakasha DG, Veeresha P, Baskonus HM (2019) Analysis of the dynamics of hepatitis e virus using the atangana-baleanu fractional derivative. Eur Phys J Plus 134(5):241

13. Morales-Delgado VF, Gómez-Aguilar JF, Saad K, Escobar Jiménez RF (2019) Application of the caputo-fabrizio and atanganabaleanu fractional derivatives to mathematical model of cancer chemotherapy effect. Math Methods Appl Sci 42(4):1167-1193

14. Uçar S, Uçar E, Özdemir N, Hammouch Z (2019) Mathematical analysis and numerical simulation for a smoking model with atangana-baleanu derivative. Chaos Solitons Fract 118:300-306

15. Kumar S, Cao J, Abdel-Aty M (2020) A novel mathematical approach of covid-19 with non-singular fractional derivative. Chaos Solitons Fract 139:110048

16. Mardani A, Hooshmandasl MR, Heydari MH, Cattani C (2018) A meshless method for solving the time fractional advectiondiffusion equation with variable coefficients. Comput Math Appl 75(1):122-133

17. Bu W, Liu X, Tang Y, Yang J (2015) Finite element multigrid method for multi-term time fractional advection diffusion equations. Int J Model Simul Sci Comput 6(1):1540001

18. Sarboland M (2018) Numerical solution of time fractional partial differential equations using multiquadric quasi-interpolation scheme. Eur J Comput Mech 27(2):89-108

19. Tian W, Deng W, Wu Y (2014) Polynomial spectral collocation method for space fractional advection-diffusion equation. Numer Methods Part Differ Equ 30(2):514-535

20. Zheng Y, Li C, Zhao Z (2010) A note on the finite element method for the space-fractional advection diffusion equation. Comput Math Appl 59(5):1718-1726

21. Shen S, Liu F, Anh V (2011) Numerical approximations and solution techniques for the space-time riesz-caputo fractional advection-diffusion equation. Numer Algorithms 56(3):383-403

22. Azin H, Mohammadi F, Heydari MH (2020) A hybrid method for solving time fractional advection-diffusion equation on unbounded space domain. Adv Diff Equ 2020(1):596

23. Ahmed N, Shah NA, Vieru D (2019) Two-dimensional advectiondiffusion process with memory and concentrated source. Symmetry 11(7):879

24. Mirza IA, Vieru D (2017) Fundamental solutions to advectiondiffusion equation with time-fractional caputo-fabrizio derivative. Comput Math Appl 73(1):1-10

25. Baleanu D, Agheli B, Al Qurashi MM (2016) Fractional advection differential equation within caputo and caputo-fabrizio derivatives. Adv Mech Eng 8(12):168781401668330

26. Rubbab Q, Mirza IA, Qureshi MZA (2016) Analytical solutions to the fractional advection-diffusion equation with time-dependent pulses on the boundary. AIP Adv 6(7):075318

27. Rubbab Q, Nazeer M, Ahmad F, Chu YM, Khan MI, Kadry S (2021) Numerical simulation of advection-diffusion equation with caputo-fabrizio time fractional derivative in cylindrical domains: applications of pseudo-spectral collocation method. Alexandria Eng J 60(1):1731-1738

28. Korpinar Z, Inc M, Baleanu D, Bayram M (2019) Theory and application for the time fractional gardner equation with mittagleffler kernel. J Taibah Univ Sci 13(1):813-819

29. Owolabi KM (2018) Numerical approach to fractional blow-up equations with atangana-baleanu derivative in riemann-liouville sense. Math Model Nat Phenomena 13(1):7

30. Owolabi KM (2018) Analysis and numerical simulation of multicomponent system with atangana-baleanu fractional derivative. Chaos Solitons Fract 115:127-134
31. Kumar D, Singh J, Baleanu D (2020) On the analysis of vibration equation involving a fractional derivative with mittag-leffler law. Math Methods Appl Sci 43(1):443-457

32. Hosseininia M, Heydari MH (2019) Meshfree moving least squares method for nonlinear variable-order time fractional $2 \mathrm{~d}$ telegraph equation involving mittag-leffler non-singular kernel. Chaos Solitons Fract 127:389-399

33. Inc M, Yusuf A, Aliyu AI, Baleanu D (2018) Investigation of the logarithmic-kdv equation involving mittag-leffler type kernel with atangana-baleanu derivative. Phys A Stat Mech Appl 506:520-531

34. Bas E, Ozarslan R (2018) Real world applications of fractional models by atangana-baleanu fractional derivative. Chaos Solitons Fract 116:121-125

35. Akgül A (2018) A novel method for a fractional derivative with non-local and non-singular kernel. Chaos Solitons Fract 114:478-482

36. Akgül A, Modanli M (2019) Crank-nicholson difference method and reproducing kernel function for third order fractional differential equations in the sense of atangana-baleanu caputo derivative. Chaos Solitons Fract 127:10-16

37. Attia N, Akgül A, Seba D, Nour A (2020) On solutions of timefractional advection-diffusion equation. Numer Methods Part Differ Equ 1-28

38. Yaseen M, Abbas M, Ahmad B (2021) Numerical simulation of the nonlinear generalized time-fractional klein-gordon equation using cubic trigonometric b-spline functions. Math Methods Appl Sci 44(1):901-916

39. Abbas M, Iqbal MK, Zafar B, Zin SBM (2019) New cubic b-spline approximations for solving non-linear third-order korteweg-de vries equation. Indian J Sci Technol 12(6):1-9

40. Khalid N, Abbas M, Iqbal MK (2020) A numerical investigation of caputo time fractional allen-cahn equation using redefined cubic b-spline functions. Adv Differ Equ 158:1-22

41. Akram T, Abbas M, Ali A (2021) A numerical study on time fractional fisher equation using an extended cubic b-spline approximation. J Math Comput Sci 22(1):85-96

42. Akram T, Abbas M, Ismail AI, Ali NHM, Baleanu D (2019) Extended cubic b-splines in the numerical solution of time fractional telegraph equation. Adv Differ Equ 2019(1):365

43. Iqbal MK, Abbas M, Nazir T, Ali N (2020) Application of new quintic polynomial $b$-spline approximation for numerical investigation of kuramoto-sivashinsky equation. Adv Differ Equ 1-21:558

44. Khalid N, Abbas M, Iqbal MK, Singh J, Ismail AIM (2020) A computational approach for solving time fractional differential equation via spline functions. Alexandria Eng J 59(5):3061-3078

45. Poulin JR (2020) Calculating infinite series using Parsevals identity (master thesis), The University of Maine, Orono

46. Yadav S, Pandey RK, Shukla AK (2019) Numerical approximations of atangana-baleanu caputo derivative and its application. Chaos Solitons Fract 118:58-64

47. Boyce WE, Diprima RC, Meade DB (1992) Elementary differential equations and boundary value problems, vol 9. Wiley, New York

48. Kadalbajoo MK, Arora P (2009) B-spline collocation method for the singular-perturbation problem using artificial viscosity. Comput Math Appl 57(4):650-663

49. Hall C (1968) On error bounds for spline interpolation. J Approx Theory 1(2):209-218

50. de Boor C (1968) On the convergence of odd-degree spline interpolation. J Approx Theory 1(4):452-463

Publisher's Note Springer Nature remains neutral with regard to jurisdictional claims in published maps and institutional affiliations. 